\section{Control of Partial Differential Equations}

\author{
Fatiha Alabau-Boussouira ${ }^{1}$, \\ Piermarco CANNARSA ${ }^{2}$ \\ ${ }^{1}$ L.M.A.M., Université de Metz, Metz, France \\ 2 Dipartimento di Matematica, Università di Roma \\ "Tor Vergata", Rome, Italy
}

\section{Article Outline \\ Glossary \\ Definition of the Subject \\ Introduction \\ Controllability \\ Stabilization \\ Optimal Control \\ Future Directions \\ Bibliography}

\section{Glossary}

$\mathbb{R}$ denotes the real line, $\mathbb{R}^{n}$ the $n$-dimensional Euclidean space, $x \cdot y$ stands for the Euclidean scalar product of $x, y \in \mathbb{R}^{n}$, and $|x|$ for the norm of $x$.

State variables quantities describing the state of a system; in this note they will be denoted by $u$; in the present setting, $u$ will be either a function defined on a subset of $\mathbb{R} \times \mathbb{R}^{n}$, or a function of time taking its values in an Hilbert space $H$.

Space domain the subset of $\mathbb{R}^{n}$ on which state variables are defined.

Partial differential equation a differential equation containing the unknown function as well as its partial derivatives.

State equation a differential equation describing the evolution of the system of interest.

Control function an external action on the state equation aimed at achieving a specific purpose; in this note, control functions they will be denoted by $f ; f$ will be used to denote either a function defined on a subset of $\mathbb{R} \times \mathbb{R}^{n}$, or a function of time taking its values in an Hilbert space $F$. If the state equation is a partial differential equation of evolution, then a control function can be:

1. distributed if it acts on the whole space domain;

2. locally distributed if it acts on a subset of the space domain;

3. boundary if it acts on the boundary of the space domain;
4. optimal if it minimizes (together with the corre- 44 sponding trajectory) a given cost;

5. feedback if it depends, in turn, on the state of the system.

Trajectory the solution of the state equation $u_{f}$ that corresponds to a given control function $f$.

Distributed parameter system a system modeled by an evolution equation on an infinite dimensional space, such as a partial differential equation or a partial integro-differential equation, or a delay equation; unlike systems described by finitely many state variables, such as the ones modeled by ordinary differential equations, the information concerning these systems is "distributed" among infinitely many parameters.

$\mathbb{1}_{A}$ denotes the characteristic function of a set $A \subset \mathbb{R}^{n}$, that is,

$$
\mathbb{1}_{A}(x)= \begin{cases}1 & x \in A \\ 0 & x \in \mathbb{R}^{n} \backslash A\end{cases}
$$

$\partial_{t}, \partial_{x_{i}}$ denote partial derivatives with respect to $t$ and $x_{i}$, respectively.

$L^{2}(\Omega)$ denotes the Lebesgue space of all real-valued square integrable functions, where functions that differ on sets of zero Lebesgue measure are identified.

$H_{0}^{1}(\Omega)$ denotes the Sobolev space of all real-valued functions which are square integrable together with their first order partial derivatives in the sense of distributions in $\Omega$, and vanish on the boundary of $\Omega$; similarly $H^{2}(\Omega)$ denotes the space of all functions which are square integrable together with their second order partial derivatives.

$H^{-1}(\Omega)$ denotes the dual of $H_{0}^{1}(\Omega)$.

$\mathcal{H}^{n-1}$ denotes the $(n-1)$-dimensional Hausdorff measure.

$H$ denotes a normed spaces over $\mathbb{R}$ with norm $\|\cdot\|$, as well as an Hilbert space with the scalar product $\langle\cdot, \cdot\rangle$ and norm $\|\cdot\|$.

$L^{2}(0, T ; H)$ is the space of all square integrable functions $f:[0, T] \rightarrow H ; C([0, T] ; H)$ (continuous functions) and $H^{1}(0, T ; H)$ (Sobolev functions) are similarly defined.

Given Hilbert spaces $F$ and $H, \mathcal{L}(F, H)$ denotes the (Banach) space of all bounded linear operators $\Lambda: F \rightarrow H$ with norm $\|\Lambda\|=\sup _{\|x\|=1}\|\Lambda x\|$ (when $F=H$, we use the abbreviated notation $\mathcal{L}(H)) ; \Lambda^{*}: H \rightarrow F$ denotes the adjoint of $\Lambda$ given by $\left\langle\Lambda^{*} u, \phi\right\rangle=\langle u, \Lambda \phi\rangle$ for all $u \in H, \phi \in F$.

Please note that the pagination is not final; in the print version an entry will in general not start on a new page. 


\section{Definition of the Subject}

Control theory (abbreviated, CT) is concerned with several ways of influencing the evolution of a given system by an external action. As such, it originated in the nineteenth century, when people started to use mathematics to analyze the perfomance of mechanical systems, even though its roots can be traced back to the calculus of variation, a discipline that is certainly much older. Since the second half of the twentieth century its study was pursued intensively to address problems in aerospace engineering, and then economics and life sciences. At the beginning, CT was applied to systems modeled by ordinary differential equations (abbreviated, ODE). It was a couple of decades after the birth of CT-in the late sixties, early seventies-that the first attempts to control models described by a partial differential equation (abbreviated, PDE) were made. The need for such a passage was unquestionable: too many interesting applications, from diffusion phenomena to elasticity models, from fluid dynamics to traffic flows on networks and systems biology, can be modeled by a PDE.

Because of its peculiar nature, control of PDE's is a rather deep and technical subject: it requires a good knowledge of PDE theory, a field of enormous interest in its own right, as well as familiarity with the basic aspects of CT for ODE's. On the other hand, the effort put into this research direction has been really intensive. Mathematicians and engineers have worked together in the construction of this theory: the results-from the stabilization of flexible structures to the control of turbulent flows-have been absolutely spectacular.

Among those who developed this subject are A. V. Balakrishnan, H. Fattorini, J. L. Lions, and D. L. Russell, but many more have given fundamental contributions.

\section{Introduction}

The basic examples of controlled partial differential equations are essentially two: the heat equation and the and the wave equation. In a bounded open domain $\Omega \subset \mathbb{R}^{n}$ with sufficiently smooth boundary $\Gamma$ the heat equation

$$
\partial_{t} u=\Delta u+f \quad \text { in } Q_{T} \doteq(0, T) \times \Omega
$$

describes the evolution in time of the temperature $u(t, x)$ at any point $x$ of the body $\Omega$. The term $\Delta u=\partial_{x_{1}}^{2} u+\cdots+$ $\partial_{x_{n}}^{2} u$, called the Laplacian of $u$, accounts for heat diffusion in $\Omega$, whereas the additive term $f$ represents a heat source. In order to solve the above equation uniquely one needs to add further data, such as the initial distribution $u_{0}$ and the temperature of the boundary surface $\Gamma$ of $\Omega$. The fact that, for any given data $u_{0} \in L^{2}(\Omega)$ and $f \in L^{2}\left(Q_{T}\right)$ Eq. (1) admits a unique weak solution $u_{f}$ satisfying the boundary condition

$$
u=0 \quad \text { on } \quad \Sigma_{T} \doteq(0, T) \times \Gamma
$$

and the initial condition

$$
u(0, x)=u_{0}(x) \quad \forall x=\left(x_{1}, \ldots, x_{n}\right) \in \Omega
$$

is well-known. So is the maximal regularity result ensuring that

$$
\begin{array}{r}
u_{f} \in H^{1}\left(0, T ; L^{2}(\Omega)\right) \cap C\left([0, T] ; H_{0}^{1}(\Omega)\right) \\
\cap L^{2}\left(0, T ; H^{2}(\Omega)\right)
\end{array}
$$

whenever $u_{0} \in H_{0}^{1}(\Omega)$. If problem (1)-(3) possesses a unique solution which depends continuously on data, then we say that the problem is well-posed.

Similarly, the wave equation

$$
\partial_{t}^{2} u=\Delta u+f \quad \text { in } \quad Q_{T}
$$

describes the vibration of an elastic membrane (when $n=2)$ subject to a force $f$. Here, $u(t, x)$ denotes the displacement of the membrane at time $t$ in $x$. The initial condition now concerns both initial displacement and velocity:

$$
\forall x \in \Omega \quad\left\{\begin{array}{l}
u(0, x)=u_{0}(x) \\
\partial_{t} u(0, x)=u_{1}(x) .
\end{array}\right.
$$

It is useful to treat the above problems as a first order evolution equation in a Hilbert space $H$

$$
u^{\prime}(t)=A u(t)+B f(t) \quad t \in(0, T),
$$

where $f(t)$ takes its valued in another Hilbert space $F$, and $B \in \mathcal{L}(F, H)$. In this abstract set-up, the fact that (7) is related to a PDE translates into that the closed linear operator $A$ is not defined on the whole space but only on a (dense) subspace $D(A) \subset H$, called the domain of $A$; such a property is often referred to as the unboundedness of $A$.

For instance, in the case of the heat equation (1), $H=L^{2}(\Omega)=F$, and $A$ is defined as

$$
\left\{\begin{array}{l}
D(A)=H^{2}(\Omega) \cap H_{0}^{1}(\Omega) \\
A u=\Delta u, \quad \forall u \in D(A),
\end{array}\right.
$$

whereas $B=I$.

As for the wave equation, since it is a second order differential equation with respect to $t$, the Hilbert space $H$ should be given by the product $H_{0}^{1}(\Omega) \times L^{2}(\Omega)$. Then, problem (5) is turned into the first order equation

$$
U^{\prime}(t)=\mathcal{A} U(t)+B f(t) \quad t \in(0, T),
$$


where

$$
U=\left(\begin{array}{l}
u \\
v
\end{array}\right), \quad B=\left(\begin{array}{c}
0 \\
I
\end{array}\right), \quad F=L^{2}(\Omega) .
$$

Accordingly, $\mathcal{A}: D(\mathcal{A}) \subset H \rightarrow H$ is given by

$$
\left\{\begin{array}{l}
D(\mathcal{A})=\left(H^{2}(\Omega) \cap H_{0}^{1}(\Omega)\right) \times H_{0}^{1}(\Omega) \\
\mathcal{A} U=\left(\begin{array}{ll}
0 & I \\
A & 0
\end{array}\right) U=\left(\begin{array}{c}
v \\
A u
\end{array}\right) \quad \forall U \in D(\mathcal{A}),
\end{array}\right.
$$

where $A$ is taken as in (8).

Another advantage of the abstract formulation (7) is the possibility of considering locally distributed or boundary source terms. For instance, one can reduce to the same set-up the equation

$$
\partial_{t} u=\Delta u+\mathbb{1}_{\omega} f \quad \text { in } Q_{T},
$$

where $\mathbb{1}_{\omega}$ denotes the characteristic function of an open set $\omega \subset \Omega$, or the nonhomogeneus boundary condition of Dirichlet type

$$
u=f \quad \text { on } \Sigma_{T},
$$

or Neumann type

$$
\frac{\partial u}{\partial v}=f \quad \text { on } \Sigma_{T},
$$

where $v$ is the outward unit normal to $\Gamma$. For Eq. (9), $B$ reduces to multiplication by $\mathbb{1}_{\omega}$-a bounded operator on $L^{2}(\Omega)$; conditions (10) and (11) can also be associated to suitable linear operators $B$-which, in this case, turn out to be unbounded. Similar considerations can be adapted to the wave equations (5) and to more general problems.

Having an efficient way to represent a source term is essential in control theory, where such a term is regarded as an external action, the control function, exercised on the state variable $u$ for a purpose, of which there are two main kinds:

- positional: $u(t)$ is to approach a given target in $X$, or attain it exactly at a given time $t>0$;

- optimal: the pair $(u, f)$ is to minimize a given functional.

The first criterion leads to approximate or exact controllability problems in time $t$, as well as to stabilization problems as $t \rightarrow \infty$. Here, the main tools will be provided by certain estimates for partial differential operators that allow to study the states that can be attained by the solution of a given controlled equation. These issues will be addressed in Sects. "Controllability" and "Stabilization" for linear evolution equations. Applications to the heat and wave equations will be discussed in the same sections.

On the other hand, optimal control problems require analyzing the typical issues of optimizations: existence results, necessary conditions for optimality, sufficient conditions, robustness. Here, the typical problem that has been successfully studied is the Linear Quadratic Regulator that will be discussed in Sect. "Linear Quadratic Optimal Control".

Control problems for nonlinear partial differential equations are extremely interesting but harder to deal with, so the literature is less rich in results and techniques. Nevertheless, among the problems that received great attention are those of fluid dynamics, specifically the Euler equations

$$
\partial_{t} u+(u \cdot \nabla) u+\nabla p=0
$$

and the Navier-Stokes equations

$$
\partial_{t} u-\mu \Delta u+(u \cdot \nabla) u+\nabla p=0
$$

subject to a boundary control and to the incompressibility condition $\operatorname{div} u=0$.

\section{Controllability}

We now proceed to introduce the main notions of controllability for the evolution equation (7). Later on in this section we will give interpretations for the heat and wave equations.

In a given Hilbert space $H$, with scalar product $\langle\cdot, \cdot\rangle$ and norm $\|\cdot\|$, let

$$
A: D(A) \subset H \rightarrow H
$$

be the infinitesimal generator of a strongly continuous semigroup $e^{t A}, t \geq 0$, of bounded linear operators on $X$. Intuitively, this amounts to saying that $u(t) \doteq \mathrm{e}^{t A} u_{0}$ is the unique solution of the Cauchy problem

$$
\left\{\begin{array}{l}
u^{\prime}(t)=A u(t) \quad t \geq 0 \\
u(0)=u_{0}
\end{array}\right.
$$

in the classical sense for $u_{0} \in D(A)$, and in a suitable generalized sense for all $u_{0} \in H$. Necessary and sufficient conditions in order for an unbounded operator $A$ to be the infinitesimal generator of a strongly continuous semigroup are given by the celebrated Hille-Yosida Theorem, see, e.g. [99] and [55]. 


\section{Abstract Evolution Equations}

Let $F$ be another Hilbert space (with scalar product and norm denoted by the same symbols as for $H$ ), the socalled control space, and let $B: F \rightarrow H$ be a linear operator, that we will assume to be bounded for the time being. Then, given $T>0$ and $u_{0} \in H$, for all $f \in L^{2}(0, T ; F)$ the Cauchy problem

$$
\left\{\begin{array}{l}
u^{\prime}(t)=A u(t)+B f(t) \quad t \geq 0 \\
u(0)=u_{0}
\end{array}\right.
$$

has a unique mild solution $u_{f} \in C([0, T] ; H)$ given by

$$
u_{f}(t)=\mathrm{e}^{t A} u_{0}+\int_{0}^{t} \mathrm{e}^{(t-s) A} B f(s) \quad \forall t \geq 0
$$

Note 1 Boundary control problems can be reduced to the same abstract form as above. In this case, however, $B$ in (12) turns out to be an unbounded operator related to suitable fractional powers of $-A$, see, e.g., [22].

For any $t \geq 0$ let us denote by $\Lambda_{t}: L^{2}(0, t ; F) \rightarrow H$ the bounded linear operator

$$
\Lambda_{t} f=\int_{0}^{t} \mathrm{e}^{(t-s) A} B f(s) \mathrm{d} s \quad \forall f \in L^{2}(0, t ; F) .
$$

The attainable (or reachable) set from $u_{0}$ at time $t$, $\mathcal{A}\left(u_{0}, t\right)$ is the set of all points in $H$ of the form $u_{f}(t)$ for some control function $f$, that is

$$
\mathcal{A}\left(u_{0}, t\right) \doteq \mathrm{e}^{t A} u_{0}+\Lambda_{t} L^{2}(0, t ; F) .
$$

We introduce below the main notions of controllability for (7). Let $T>0$.

Definition 1 System (7) is said to be:

- exactly controllable in time $T$ if $\mathcal{A}\left(u_{0}, T\right)=H$ for all $u_{0} \in H$, that is, if for all $u_{0}, u_{1} \in H$ there is a control function $f \in L^{2}(0, T ; F)$ such that $u_{f}(T)=u_{1}$;

- null controllable in time $T$ if $0 \in \mathcal{A}\left(u_{0}, T\right)$ for all $u_{0} \in H$, that is, if for all $u_{0} \in H$ there is a control function $f \in L^{2}(0, T ; F)$ such that $u_{f}(T)=0$;

- approximately controllable in time $T$ if $\mathcal{A}\left(u_{0}, T\right)$ is dense in $H$ for all $u_{0} \in H$, that is, if for all $u_{0}, u_{1} \in H$ and for any $\varepsilon>0$ there is a control function $f \in L^{2}(0, T ; F)$ such that $\left\|u_{f}(T)-u_{1}\right\|<\varepsilon$.

Clearly, if a system is exactly controllable in time $T$, then it is also null and approximately controllable in time $T$. Although these last two notions of controllability are strictly weaker than strong controllability, for specific problems-like when $A$ generates a strongly continuous 292 group-some of them may coincide.

Since controllability properties concern, ultimately, the range of the linear operator $\Lambda_{T}$ defined in (14), it is not surprising that they can be characterized in terms of the adjoint operator $\Lambda_{T}^{*}: H \rightarrow L^{2}(0, T ; F)$, which is defined by

$$
\begin{aligned}
\int_{0}^{T}\left\langle\Lambda_{T}^{*} u(s), f(s)\right\rangle \mathrm{d} s & =\left\langle u_{0}, \Lambda_{T} f\right\rangle \\
& \forall u \in H, \quad \forall f \in L^{2}(0, T ; F) .
\end{aligned}
$$

Such a characterization is the object of the following theorem. Notice that the above identity and (14) yield

$$
\Lambda_{T}^{*} u(s)=B^{*} \mathrm{e}^{(T-s) A^{*}} u \quad \forall s \in[0, T] .
$$

Theorem 1 System (7) is:

- exactly controllable in time $T$ if and only if there is a constant $C>0$ such that

$$
\int_{0}^{T}\left\|B^{*} \mathrm{e}^{t A^{*}} u\right\|^{2} \mathrm{~d} t \geq C\|u\|^{2} \quad \forall u \in H ;
$$

- null controllable in time $T$ if and only if there is a constant $C>0$ such that

$$
\int_{0}^{T}\left\|B^{*} \mathrm{e}^{t A^{*}} u\right\|^{2} \mathrm{~d} t \geq C\left\|\mathrm{e}^{T A^{*}} u\right\|^{2} \quad \forall u \in H ;
$$

- approximately controllable in time $T$ if and only if, for every $u \in H$,

$$
B^{*} \mathrm{e}^{t A^{*}} u=0 \quad t \in[0, T] \text { a.e. } \Longrightarrow u=0 .
$$

To benefit the reader who is more familiar with optimization theory than abstract functional analysis, let us explain, by a variational argument, why estimate (16) implies null controllability. Consider, for every $\varepsilon>0$, the penalized problem

$$
\min \left\{J_{\varepsilon}(f): f \in L^{2}(0, T ; H)\right\},
$$

where

$$
\begin{aligned}
J_{\varepsilon}(f)=\frac{1}{2} \int_{0}^{T}\|f(t)\|^{2} \mathrm{~d} t+\frac{1}{2 \varepsilon}\left\|u_{f}(T)\right\|^{2} & \\
& \forall f \in L^{2}(0, T ; H) .
\end{aligned}
$$

Since $J_{\varepsilon}$ is strictly convex, it admits a unique minimum point $f_{\varepsilon}$. Set $u_{\varepsilon}=u_{f_{\varepsilon}}$. Recalling (13) we have, By Fermat's rule,

$$
\begin{aligned}
0=J_{\varepsilon}^{\prime}\left(f_{\varepsilon}\right) g & =\int_{0}^{T}\left\langle f_{\varepsilon}(t), g(t)\right\rangle \mathrm{d} t \\
& +\frac{1}{\varepsilon}\left\langle u_{\varepsilon}(T), \Lambda_{T} g\right\rangle \quad \forall g \in L^{2}(0, T ; H) .
\end{aligned}
$$

303 
Therefore, passing to the adjoint of $\Lambda_{T}$,

$$
\begin{aligned}
\int_{0}^{T}\left\langle f_{\varepsilon}(t)+\frac{1}{\varepsilon}\left(\Lambda_{T}^{*} u_{\mathcal{\varepsilon}}(T)\right)(t), g(t)\right\rangle \mathrm{d} t & =0 \\
\forall g & \in L^{2}(0, T ; H),
\end{aligned}
$$

whence, owing to (14),

$$
f_{\varepsilon}(t)=-\frac{1}{\varepsilon}\left(\Lambda_{T}^{*} u_{\varepsilon}(T)\right)(t)=-B^{*} v_{\varepsilon}(t)
$$

$$
\forall t \in[0, T],
$$

where $v_{\varepsilon}(t) \doteq \frac{1}{\varepsilon} \mathrm{e}^{(T-t) A^{*}} u_{\varepsilon}(T)$ is the solution of the dual problem

$$
\left\{\begin{array}{l}
v^{\prime}+A^{*} v=0 \quad t \in[0, T] \\
v(T)=\frac{1}{\varepsilon} u_{\varepsilon}(T)
\end{array}\right.
$$

It turns out that

$$
\begin{aligned}
& \frac{1}{2} \int_{0}^{T}\left\|f_{\varepsilon}(t)\right\|^{2} \mathrm{~d} t+\frac{1}{\varepsilon}\left\|u_{\varepsilon}(T)\right\|^{2} \leq C\left\|u_{0}\right\|^{2} \\
& \forall \varepsilon>0
\end{aligned}
$$

for some positive constant $C$. Indeed, observe that, in view of (19),

$$
\begin{cases}\left\langle u_{\varepsilon}^{\prime}-A u_{\varepsilon}+B B^{*} v_{\varepsilon}, v_{\varepsilon}\right\rangle=0, & u_{\varepsilon}(0)=u_{0} \\ \left\langle v_{\varepsilon}^{\prime}+A^{*} v_{\varepsilon}, u_{\varepsilon}\right\rangle=0, & v_{\varepsilon}(T)=\frac{1}{\varepsilon} u_{\varepsilon}(T) .\end{cases}
$$

So,

$$
\int_{0}^{T}\left[\frac{\mathrm{d}}{\mathrm{d} t}\left\langle u_{\varepsilon}, v_{\varepsilon}\right\rangle+\left\|B^{*} v_{\varepsilon}\right\|^{2}\right] d t=0,
$$

whence

$$
\frac{1}{\varepsilon}\left\|u_{\varepsilon}(T)\right\|^{2}+\int_{0}^{T}\left\|B^{*} v_{\varepsilon}\right\|^{2} \mathrm{~d} t=\left\langle u_{0}, v_{\varepsilon}(0)\right\rangle .
$$

Now, apply estimate (16) with $u=\frac{u_{\varepsilon}(T)}{\varepsilon}$ and note that $v_{\varepsilon}(T-t)=\mathrm{e}^{t A^{*}} \frac{u_{\varepsilon}(T)}{\varepsilon}$ to obtain

$$
\int_{0}^{T}\left\|B^{*} v_{\varepsilon}(t)\right\|^{2} \mathrm{~d} t \geq C\left\|v_{\varepsilon}(0)\right\|^{2}
$$

for some positive constant $C$. Hence, (20) follows from (21) and (19).

Finally, from (20) one deduces the existence of a weakly convergent subsequence $f_{\varepsilon_{j}}$ in $L^{2}(0, T ; F)$. Then, called $f_{0}$ the weak limit of $f_{\varepsilon_{j}}, u_{\varepsilon_{j}}(t) \rightarrow u_{f_{0}}(t)$ for all $t \in[0, T]$. So, owing to $(20), u_{f_{0}}(T)=0$.

\section{Heat Equation}

It is not hard to see that the heat equation (9) with Dirichlet boundary conditions (2) fails to be exactly controllable. On the other hand, one can show that it is null controllable in any time $T>0$, hence approximately controllable. Let $\omega$ be an open subset of $\Omega$ such that $\bar{\omega} \subset \Omega$.

Taking

$$
H=L^{2}(\Omega)=F, \quad B f=\mathbb{1}_{\omega} f \quad \forall f \in L^{2}(\Omega)
$$

and $A$ as in (8), one obtains that, for any $u_{0} \in L^{2}(\Omega)$ and $f \in L^{2}\left(Q_{T}\right)$, the initial-boundary value problem

$$
\begin{cases}\partial_{t} u=\Delta u+\mathbb{1}_{\omega} f & \text { in } Q_{T} \\ u=0 & \text { on } \Sigma_{T} \\ u(0, x)=u_{0}(x) & x \in \Omega\end{cases}
$$

has a unique mild solution $u_{f} \in C\left([0, T] ; L^{2}(\Omega)\right)$. Moreover, multiplying both sides of equation (9) by $u$ and integrating by parts, it is easy to see that

$$
\partial_{x_{i}} u \in L^{2}\left(Q_{T}\right) \quad \forall i=1, \ldots, n .
$$

Notice that the above property already suffices to explain why the heat equation cannot be exactly controllable: it is impossible to attain a state $u_{1} \in L^{2}(\Omega)$ which fails to satisfy (23).

On the other hand, null controllability holds true in any positive time.

Theorem 2 Let $T>0$ and let $\omega$ be an open subset of $\Omega$ such that $\bar{\omega} \subset \Omega$. Then the heat equation (9) with homogeneous Dirichlet boundary conditions is null controllable in time $T$, i.e., for every initial condition $u_{0} \in L^{2}(\Omega)$ there is a control function $f \in L^{2}\left(Q_{T}\right)$ such that the solution $u_{f}$ of (22) satisfies $u_{f}(T, \cdot) \equiv 0$. Moreover,

$$
\iint_{Q_{T}}|f|^{2} \mathrm{~d} x \mathrm{~d} t \leq C_{T} \int_{\Omega}\left|u_{0}\right|^{2} \mathrm{~d} x
$$

for some positive constant $C_{T}$.

The above property is a consequence of the abstract result in Theorem 1 and of concrete estimates for solutions of parabolic equations. Indeed, in order to apply Theorem 1 one has to translate (16) into an estimate for the heat operator. Now, observing that both $A$ and $B$ are self-adjoint, one promptly realizes that (16) reduces to

$$
\int_{0}^{T} \int_{\omega}|v(t, x)|^{2} \mathrm{~d} x \mathrm{~d} t \geq C \int_{\Omega}|v(T, x)|^{2} \mathrm{~d} x
$$

for every solution $v$ of the problem

$$
\begin{cases}\partial_{t} v=\Delta v & \text { in } Q_{T} \\ v=0 & \text { on } \Sigma_{T} .\end{cases}
$$




$$
{ }_{441} \quad \frac{\partial \phi}{\partial v}(x)=\nabla \phi(x) \cdot v(x)
$$
ally of the form $C^{2}(\bar{\Omega})$ such that

$$
\nabla \phi(x) \neq 0 \quad \forall x \in \bar{\Omega},
$$

and

Note that $\Gamma$ at a point $x \in \Gamma$, and by
Estimate (24) is called an observability inequality for the heat operator for obvious reasons: problem (25) is not well-posed since the initial condition is missing. Nevertheless, if, "observing" a solution $v$ of such a problem on the "small" cylinder $(0, T) \times \omega$, you find that it vanishes, then you can conclude that $v(T, \cdot) \equiv 0$ in the whole domain $\Omega$. Thus, $v(0, \cdot) \equiv 0$ by backward uniqueness.

In conclusion, as elegant as the abstract approach to null controllability may be, one is confronted by the difficult task of proving observability estimates. In fact, for the heat operator there are several ways to prove inequality (24). One of the most powerful, basically due to Fursikov and Imanuvilov [65], relies on global Carleman estimates. Compared to other methods that can be used to derive observability, such a technique has the advantage of applying to second order parabolic operators with variable coefficients, as well as to more general operators.

Global Carleman estimates are a priori estimates in weighted norms for solutions of the problem

$$
\begin{cases}\partial_{t} v=\Delta v+f & \text { in } Q_{T} \\ v=0 & \text { on } \Sigma_{T} .\end{cases}
$$

regardless of initial conditions. The weight function is usu-

$$
\psi_{r}(t, x) \doteq \theta(t)\left(\mathrm{e}^{2 r\|\phi\|_{\infty}, \Omega}-\mathrm{e}^{r \phi(x)}\right) \quad(t, x) \in Q_{T},
$$

where $r$ is a positive constant, $\phi$ is a given function in

$$
\theta(t) \doteq \frac{1}{t(T-t)} \quad 0<t<T
$$

$$
\theta>0, \quad \theta(t) \rightarrow \infty \quad t \rightarrow 0, T
$$

$$
\psi_{r}>0, \quad \psi_{r}(t, x) \rightarrow \infty \quad t \downarrow 0, t \uparrow T .
$$

Using the above notations, a typical global Carleman estimate for the heat operator is the following result obtained in [65]. Let us denote by $v(x)$ the outword unit normal to

the normal derivative of $\phi$ at $x$.
Theorem 3 Let $\Omega$ be a bounded domain of $\mathbb{R}^{n}$ with boundary of class $C^{2}$, let $f \in L^{2}\left(Q_{T}\right)$, and let $\phi$ be a function satisfying (28). Let $v$ be a solution of (26). Then there are positive constants $r, s_{0}$ and $C$ such that, for any $s>s_{0}$,

$$
\begin{aligned}
& s^{3} \iint_{Q_{T}} \theta^{3}(t)|v(t, x)|^{2} \mathrm{e}^{-2 s \psi_{r}} \mathrm{~d} x \mathrm{~d} t \\
& \leq C \iint_{Q_{T}}|f(t, x)|^{2} \mathrm{e}^{-2 s \psi_{r}} \mathrm{~d} x \mathrm{~d} t \\
& +C s \int_{0}^{T} \theta(t) \mathrm{d} t \\
& \quad \times \int_{\Gamma} \frac{\partial \phi}{\partial v}(x)\left|\frac{\partial v}{\partial v}(t, x)\right|^{2} \mathrm{e}^{-2 s \psi_{r}} \mathrm{~d} \mathcal{H}^{n-1}(x)
\end{aligned}
$$

It is worth underlying that, thanks to the singular behavior of $\theta$ near 0 and $T$, the above result is independent of the initial value of $v$. Therefore, it can be applied, indifferently, to any solution of (26) as well as to any solution of the backward problem

$$
\begin{cases}\partial_{t} v+\Delta v=f & \text { in } Q_{T} \\ v=0 & \text { on } \Sigma_{T} .\end{cases}
$$

Moreover, inequality (29) can be completed adding first and second order terms to its right-hand side, each with its own adapted power of $s$ and $\theta$.

Instead of trying to sketch the proof of Theorem 3, which would go beyond the scopes of this note, it is interesting to explain how it can be used to recover the observability inequality (24), which is what is needed to show that the heat equation is null controllable. The reasoning-not completely straightforward-is based on the following topological lemma, proved in [65].

Lemma 1 Let $\Omega \subset \mathbb{R}^{n}$ be a bounded domain with boundary $\Gamma$ of class $C^{k}$, for some $k \geq 2$, and let $\omega \subset \Omega$ be an open set such that $\bar{\omega} \subset \Omega$.

Then there is function $\phi \in C^{k}(\bar{\Omega})$ such that

$$
\begin{cases}\text { (i) } & \phi(x)=0 \text { and } \frac{\partial \phi}{\partial v}(x)<0 \quad \forall x \in \Gamma \\ \text { (ii }) & \{x \in \Omega \mid \nabla \phi(x)=0\} \subset \omega .\end{cases}
$$

Now, given a solution $v$ of (25) and an open set $\omega$ such that $\bar{\omega} \subset \Omega$, let $\omega^{\prime} \subset \subset \omega^{\prime \prime} \subset \subset \omega$ be subdomains with smooth boundary. Then the above lemma ensures the existence of a function $\phi$ such that . 45 .

\section{.}

.
. .

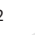
.

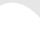


"Localizing" problem (25) onto $\Omega^{\prime} \doteq \Omega \backslash \omega^{\prime}$ by a cutoff parabolic PDE's)

$0 \leq \eta \leq 1, \quad \eta \equiv 1 \quad$ on $\quad \mathbb{R}^{n} \backslash \omega^{\prime \prime}, \quad \eta \equiv 0 \quad$ on $\quad \omega^{\prime}$,

$\int_{0}^{T} \mathrm{~d} t \int_{\omega^{\prime \prime} \backslash \omega^{\prime}}|\nabla v|^{2} \mathrm{e}^{-2 s \psi_{r}} \mathrm{~d} x$

that is, taking $w=\eta v$, gives

$$
\leq C \int_{0}^{T} \mathrm{~d} t \int_{\omega}|v|^{2} \mathrm{e}^{-2 s \psi_{r}} \mathrm{~d} x,
$$

to conclude that

$$
\int_{T / 3}^{2 T / 3} \mathrm{~d} t \int_{\Omega \backslash \omega}|v|^{2} \mathrm{~d} x \leq C \int_{0}^{T} \mathrm{~d} t \int_{\omega}|v|^{2} \mathrm{~d} x
$$

or

$$
\int_{T / 3}^{2 T / 3} \mathrm{~d} t \int_{\Omega}|v|^{2} \mathrm{~d} x \leq(1+C) \int_{0}^{T} \mathrm{~d} t \int_{\omega}|v|^{2} \mathrm{~d} x
$$

for some constant $C$. Then, the dissipativity of the heat op- $\quad 518$ erator (that is, the fact that $\int_{\Omega}|v(t, x)|^{2} \mathrm{~d} x$ is decreasing $\quad 519$ with respect to $t$ ) implies that

$$
\begin{aligned}
\int_{\Omega} v^{2}(T, x) \mathrm{d} x & \leq \frac{3}{T} \int_{T / 3}^{2 T / 3} \mathrm{~d} t \int_{\Omega} v^{2}(t, x) \mathrm{d} x \\
& \leq(1+C) \frac{3}{T} \int_{0}^{T} \mathrm{~d} t \int_{\omega} v^{2}(t, x) \mathrm{d} x
\end{aligned}
$$

which is exactly (24).

\section{Wave Equation}

Compared to the heat equation, the wave equation (5) exhibits a quite different behavior from the point of view of exact controllability. Indeed, on the one hand, there is no obstruction to exact controllability since no regularizing effect is connected with wave propagation. On the other hand, due to the finite speed of propagation, exact controllability cannot be expected to hold true in arbitrary time, as null controllability does for the heat equation.

In fact, a typical result that holds true for the wave equation is the following, where a boundary control of Dirichlet type acts on a part $\Gamma_{1} \subset \Gamma$, while homogeneous boundary conditions are imposed on $\Gamma_{0}=\Gamma \backslash \Gamma_{1}$ :

$$
\begin{cases}\partial_{t}^{2} u=\Delta u & \text { in } Q_{T} \\ u=f \mathbb{1}_{\Gamma_{1}} & \text { on } \Sigma_{T} \\ u(0, x)=u_{0}(x), \partial_{t} u(0, x)=u_{1}(x) & x \in \Omega\end{cases}
$$

Observe that problem (32) is well-posed taking

$$
u_{0} \in L^{2}(\Omega), \quad u_{1} \in H^{-1}(\Omega)
$$$$
f \in L^{2}\left(0, T ; L^{2}(\Gamma)\right)
$$

equality (a well-known estimate for solution of elliptic and
and $T_{0}=T / 3, T_{1}=2 T / 3$ and use Caccioppolis

$u \in C\left([0, T] ; L^{2}(\Omega)\right) \cap C^{1}\left([0, T] ; H^{-1}(\Omega)\right)$. 
Theorem 4 Let $\Omega$ be a bounded domain of $\mathbb{R}^{n}$ with boundary of class $C^{2}$ and suppose that, for some point $x_{0} \in \mathbb{R}^{n}$,

$$
\begin{cases}\left(x-x_{0}\right) \cdot v(x)>0 & \forall x \in \Gamma_{1} \\ \left(x-x_{0}\right) \cdot v(x) \leq 0 & \forall x \in \Gamma_{0} .\end{cases}
$$

Let

$$
R=\sup _{x \in \Omega}\left|x-x_{0}\right| .
$$

If $T>2 R$, then, for all $\left(u_{0}, u_{1}\right),\left(v_{0}, v_{1}\right) \in L^{2}(\Omega) \times H^{-1}(\Omega)$ there is a control function $f \in L^{2}\left(0, T ; L^{2}(\Gamma)\right)$ such that the solution $u_{f}$ of (32) satisfies

$$
u_{f}(T, x)=v_{0}(x), \quad \partial_{t} u_{f}(T, x)=v_{1}(x) .
$$

As we saw for abstract evolution equations, the above exact controllability property is proved to be equivalent to an observability estimate for the dual homogeneous problem using, for instance, the Hilbert Uniqueness Method (HUM) by J.-L. Lions [86].

\section{Bibliographical Comments}

The literature on controllability of parabolic equations and related topics is so huge, that no attempt to provide a comprehensive account of it would fit within the scopes of this note. So, the following comments have to be taken as a first hint for the interested reader to pursue further bibliographical research.

The theory of exact controllability for parabolic equations was initiated by the seminal paper [58] by Fattorini and Russell. Since then, it has experienced an enormous development. Similarly, the multiplier method to obtain observability inequalities for the wave equation was developed in $[17,73,74,77,86]$. Some fundamental early contributions were surveyed by Russell [108]. The next essential progress was made in the work by Lebeau and Robbiano [83] and then by Fursikov and Imanuvilov in a series of papers. In [65] one can find an introduction to global Carleman estimates, as well as applications to the controllability of several ODE's. In particular, the presentation of this paper as for observability inequalities and Carleman estimates for the heat operator is inspired by the last monograph. General perspectives for the understanding of global Carleman estimates and their applications to unique continuation and control problems for PDE's can be found in the works by Tataru [113,114,115,116]. Usually, the above approach requires coefficients to be sufficiently smooth. Recently, however, interesting adaptations of Carleman estimates to parabolic operators with discontinuous coefficients have been obtained in [21,82].

More recently, interest has focussed on control problems for nonlinear parabolic equations. Different approaches to controllability problems have been proposed in [57] and [44]. Then, null and approximate controllability results have been improved by Fernandez-Cara and Zuazua $[61,62]$. Techniques to produce insensitizing controls have been developed in [117]. These techniques have been successfully applied to the study of Navier-Stokes equations by several authors, see e. g. [63].

Fortunately, several excellent monographs are now available to help introduce the reader to this subject. For instance, the monograph by Zabczyk [121] could serve as a clean introduction to control and stabilization for finiteand infinite-dimensional systems. Moreover, [22,50,51], as well as $[80,81]$ develop all the basic concepts of control and system theory for distributed parameter systems with special emphasis on abstract formulation. Specific references for the controllability of the wave equation by HUM can be found in [86] and [74]. More recent results related to series expansion and Ingham type methods can be found in [75]. For the control of Navier-Stokes equations the reader is referred to [64], as well as to the book by Coron [43], which contains an extremely rich collection of classical results and modern developments.

\section{Stabilization}

Stabilization of flexible structures such as beams, plates, up to antennas of satellites, or of fluids as, for instance, in aeronautics, is an important part of CT. In this approach, one wants either to derive feedback laws that will allow the system to autoregulate once they are implemented, or study the asymptotic behavior of the stabilized system i. e. determine whether convergence toward equilibrium states as times goes to infinity holds, determine its speed of convergence if necessary or study how many feedback controls are required in case of coupled systems.

Different mathematical tools have been introduced to handle such questions in the context of ODE's and then of PDE's. Stabilization of ODE's goes back to the work of Lyapunov and Lasalle. The important property is that trajectories decay along Lyapunov functions. If trajectories are relatively compact in appropriate spaces and the system is autonomous, then one can prove that trajectories converge to equilibria asymptotically. However, the construction of Lyapunov functions is not easy, in general.

This section will be concerned with some aspects of the stabilization of second order hyperbolic equations, our model problem being represented by the wave equation 
${ }_{634} \begin{cases}\partial_{t t} u-\Delta u+a(x) u_{t}=0 & \text { in } \Omega \times \mathbb{R}, \\ u=0 & \text { on } \Sigma=(0, \infty) \times \Gamma \\ \left(u, \partial_{t} u\right)(0)=\left(u^{0}, u^{1}\right) & \text { on } \Omega,\end{cases}$

with distributed damping

in a bounded domain $\Omega \subset \mathbb{R}^{n}$ with a smooth boundary $\Gamma$. For $n=2, u(t, x)$ represents the displacement of point $x$ of the membrane at time $t$. Therefore, equation (33) describes an elastic system. The energy of such a system is given by

$$
E(t)=\frac{1}{2} \int_{\Omega}\left[\left|u_{t}(t, x)\right|^{2}+|\nabla u(t, x)|^{2}\right] \mathrm{d} x .
$$

When $a \geq 0$, the feedback term $a(x) u_{t}$ models friction: it produces a loss of energy through a dissipation phenomenon. More precisely, multiplying the equation in (33) by $u_{t}$ and integrating by parts on $\Omega$, it follows that

$$
E^{\prime}(t)=-\int_{\Omega} a(x)\left|u_{t}\right|^{2} \mathrm{~d} x \leq 0, \quad \forall t \geq 0
$$

On the other hand, if $a \equiv 0$, then the system is conservative, i. e., $E(t)=E(0)$ for all $t \geq 0$.

Another well-investigated stabilization problem for the wave equation is when the feedback is localized on a part $\Gamma_{0}$ of the boundary $\Gamma$, that is,

$$
\begin{cases}\partial_{t t} u-\Delta u=0 & \text { in } \Omega \times \mathbb{R} \\ \frac{\partial u}{\partial v}+u_{t}=0 & \text { on } \Sigma_{0}=(0, \infty) \times \Gamma_{0} \\ u=0 & \text { on } \Sigma_{1}=(0, \infty) \times\left(\Gamma \backslash \Gamma_{0}\right) \\ \left(u, \partial_{t} u\right)(0)=\left(u^{0}, u^{1}\right) & \end{cases}
$$

In this case, the dissipation relation (34) takes the form

$$
E^{\prime}(t)=-\int_{\Gamma_{0}}\left|u_{t}\right|^{2} \mathrm{~d} \mathcal{H}^{n-1} \leq 0, \quad \forall t \geq 0
$$

In many a situation - such as to improve the quality of an acoustic hall-one seeks to reduce vibrations to a minimum: this is why stabilization is an important issue in CT. We note that the above system has a unique stationary solution-or, equilibrium - given by $u \equiv 0$. Stabilization theory studies all questions related to the convergence of solutions to such an equilibrium: existence of the limit, rate of convergence, different effects of nonlinearities in both displacement and velocity, effects of geometry, coupled systems, damping effects due to memory in viscoelastic materials, and so on.

System (33) is said to be:
- strongly stable if $E(t) \rightarrow 0$ as $t \rightarrow \infty$;

- (uniformly) exponentially stable if $E(t) \leq C \mathrm{e}^{-\alpha t} E(0)$ for all $t \geq 0$ and some constants $\alpha>0$ and $C \geq 0$, independent of $u^{0}, u^{1}$.

This note will focus on some of the above issues, such as geometrical aspects, nonlinear damping, indirect damping for coupled systems and memory damping.

\section{Geometrical Aspects}

A well-known property of the wave equation is the socalled finite speed of propagation, which means that, if the initial conditions $u^{0}, u^{1}$ have compact support, then the support of $u(t, \cdot)$ evolves in time at a finite speed. This explains why, for the wave equation, the geometry of $\Omega$ plays an essential role in all the issues related to control and stabilization.

The size and localization of the region in which the feedback is active is of great importance. In this paper such a region, denoted by $\omega$, is taken as a subset of $\Omega$ of positive Lebesgue measure. More precisely, $a$ is assumed to be continuous on $\bar{\Omega}$ and such that

$$
a \geq 0 \quad \text { on } \quad \Omega \text { and } a \geq a_{0} \text { on } \omega, \quad \text { (36) }
$$

for some constant $a_{0}>0$. In this case, the feedback is said to be distributed. Moreover, it is said to be globally distributed if $\omega=\Omega$ and locally distributed if $\Omega \backslash \omega$ has positive Lebesgue measure.

Two main methods have been used or developed to study stabilization, namely the multiplier method and microlocal analysis. The one that gives the sharpest results is based on microlocal analysis. It goes back to the work of Bardos, Lebeau and Rauch [17], giving geodesics sufficient conditions on the region of active control for exact controllability to hold. These conditions say that each ray of geometric optics should meet the control region. Burq and Gérard [25] showed that these results hold under weaker regularity assumptions on the domain and coefficients of the operators (see also $[26,27]$ ). These geodesics conditions are not explicit, in general, but they allow to get decay estimates of the energy under very general hypotheses.

The multiplier method is an explicit method, based on energy estimates, to derive decay rates (as well as observability and exact controllability results). For boundary control and stabilization problems it was developed in the works of several authors, such as Ho $[38,73]$, J.-L. Lions [86], Lasiecka-Triggiani, Komornik-Zuazua [76], and many others. Zuazua [123] gave an explicit geometric condition on $\omega$ for a semilinear wave equation subject to a locally distributed damping. Such a condition 
was then relaxed K. Liu [87] (see also [93]) who introduced the so-called piecewise multiplier method. Lasiecka and Triggiani $[80,81]$ introduced a sharp trace regularity method which allow to estimate boundary terms in energy estimates. There also exist intermediate results between the geodesics conditions of Bardos-Lebeau-Rauch and the multiplier method, obtained by Miller [95] using differentiable escape functions.

Zuazua's multiplier geometric condition can be described as follows. If a subset $O$ of $\bar{\Omega}$ is given, one can define an $\varepsilon$-neighborhood of $O$ in $\bar{\Omega}$ as the subset of points of $\Omega$ which are at distance at most $\varepsilon$ of $O$. Zuazua proved that if the set $\omega$ is such that there exists a point $x_{0} \in \mathbb{R}^{n}$-an observation point-for which $\omega$ contains an $\varepsilon$-neighborhood of $\Gamma\left(x^{0}\right)=\left\{x \in \partial \Omega,\left(x-x^{0}\right) \cdot v(x) \geq\right.$ $0\}$, then the energy decays exponentially. In this note, we refer to this condition as (MGC).

If $a$ vanishes for instance in a neighborhood of the two poles of a ball $\Omega$ in $\mathbb{R}^{n}$, one cannot find an observation point $x_{0}$ such that (MGC) holds. K. Liu [87] (see also [93]) introduced a piecewise multiplier method which allows to choose several observation points, and therefore to handle the above case.

Introduce disjoint lipschitzian domains $\Omega_{j}$ of $\Omega$, $j=1, \ldots, J$, and observation points $x^{j} \in \mathbb{R}^{N}, j=1, \ldots$, $J$ and define

$$
\gamma_{j}\left(x^{j}\right)=\left\{x \in \partial \Omega_{j},\left(x-x^{j}\right) \cdot v_{j}(x) \geq 0\right\}
$$

Here $v_{j}$ stands for the unit outward normal vector to the boundary of $\Omega_{j}$. Then the piecewise multiplier geometrical condition for $\omega$ is:

$$
\omega \supset \mathcal{N}_{\varepsilon}\left(\cup_{j=1}^{J} \gamma_{j}\left(x^{j}\right) \cup\left(\Omega \backslash \cup_{j=1}^{J} \Omega_{j}\right)\right)
$$

(PWMGC)

It will be denoted by (PWMGC) condition in the sequel.

Assume now that $a$ vanishes in a neighborhood of the two poles of a ball in $\mathbb{R}^{n}$. Then, one can choose two subsets $\Omega_{1}$ and $\Omega_{2}$ containing, respectively, the two regions where $a$ vanishes and apply the piecewise multiplier method with $J=2$ and with the appropriate choices of two observation points and $\varepsilon$. The multiplier method consists of integrating by parts expressions of the form

$$
\int_{t}^{T} \int_{\Omega}\left(\partial_{t}^{2} u-\Delta u+a(x) u_{t}\right) M u \mathrm{~d} x \mathrm{~d} t=0
$$

$$
\forall 0 \leq t \leq T,
$$

where $u$ stands for a (strong) solution of (33), with an appropriate choice of $M u$. Multipliers have generally the form

$$
M u=(m(x) \cdot \nabla u+c u) \psi(x),
$$

where $m$ depends on the observation points and $\psi$ is a cut-off function. Other multipliers of the form $M u=\Delta^{-1}(\beta u)$, where $\beta$ is a cut-off function and $\Delta^{-1}$ is the inverse of the Laplacian operator with homogeneous Dirichlet boundary conditions, have also be used.

The geometric conditions (MGC) or (PWMGC) serve to bound above by zero terms which cannot be controlled otherwise. One can then prove that the energy satisfies an estimate of the form

$$
\begin{aligned}
& \int_{t}^{T} E(s) \mathrm{d} s \\
& \leq c E(t)+\int_{t}^{T}\left(\int_{\Omega} a(x)\left|u_{t}\right|^{2}+\int_{\omega}\left|u_{t}\right|^{2}\right) \mathrm{d} s \\
& \forall t \geq 0 .
\end{aligned}
$$

Once this estimate is proved, one can use the dissipation relation to prove that the energy satisfies integral inequalities of Gronwall type. This is the subject of the next section.

\section{Decay Rates, Integral Inequalities} and Lyapunov Techniques

The Linear Feedback Case Using the dissipation relation (34), one has

$$
\begin{aligned}
\int_{t}^{T} \int_{\Omega} a\left|u_{t}\right|^{2} \mathrm{~d} x \mathrm{~d} s \leq \int_{t}^{T}-E^{\prime}(s) \mathrm{d} s & \leq E(t) \\
& \forall 0 \leq t \leq T .
\end{aligned}
$$

On the other hand, thanks to assumption (36) on $a$

$$
\begin{aligned}
\int_{t}^{T} \int_{\omega} u_{t}^{2} \mathrm{~d} x \mathrm{~d} s \leq \frac{1}{a_{0}} \int_{t}^{T} & \int_{\Omega} a\left|u_{t}\right|^{2} \mathrm{~d} x \mathrm{~d} s \\
& \leq \frac{1}{a_{0}} E(t) \quad \forall 0 \leq t \leq T
\end{aligned}
$$

By the above inequalities and (37), E satisfies

$$
\int_{t}^{T} E(s) \mathrm{d} s \leq c E(t), \quad \forall 0 \leq t \leq T .
$$

Since $E$ is a nonincreasing function and thanks to this integral inequality, Haraux [71] (see also Komornik [74]) proved that $E$ decays exponentially at infinity, that is

$$
E(t) \leq E(0) \exp (1-t / c), \quad \forall t \geq c .
$$

This proof is as follows. Define

$$
\phi(t)=\exp (t / c) \int_{t}^{\infty} E(s) \mathrm{d} s \quad \forall t \geq 0 .
$$
(1)

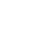
. . .

77 .

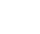


Thanks to (38) $\phi$ is nonincreasing on $[0, \infty)$, so that

$$
\phi(t) \leq \phi(0)=\int_{0}^{\infty} E(s) \mathrm{d} s .
$$

Using once again (38) with $t=0$ in this last inequality and the definition of $\phi$, one has

$$
\int_{t}^{\infty} E(s) \mathrm{d} s \leq c E(0) \exp (-t / c) \quad \forall t \geq 0 .
$$

Since $E$ is a nonnegative and nonincreasing function

$$
\begin{aligned}
c E(t) \leq \int_{t-c}^{t} E(s) \mathrm{d} s \leq & \int_{t-c}^{\infty} E(s) \mathrm{d} s \\
& \leq c E(0) \exp (-(t-c) / c),
\end{aligned}
$$

so that (39) is proved. One can remark that for $t \leq c$, $E(t) \leq E(0) \leq \exp (1-t / c)$.

An alternative method is to introduce a modified (or perturbed) energy $E_{\varepsilon}$ which is equivalent to the natural one for small values of the parameter $\varepsilon$ as in Komornik and Zuazua [76]. Then one shows that this modified energy satisfies a differential Gronwall inequality so that it decays exponentially at infinity. The exponential decay of the natural energy follows then at once. In this case, the modified energy is indeed a Lyapunov function for the PDE. The natural energy cannot be in general such a Lyapunov function due to the finite speed of propagation (consider initial data which have compact support compactly embedded in $\Omega \backslash \omega)$.

There are also very interesting approaches using the frequency domain approach, or spectral analysis such as developed by K. Liu [87] Z. Liu and S. Zheng [88]. In the sequel, we concentrate on the integral inequality method. This method has been generalized in several directions and we present in this note some results concerning extensions to

- nonlinear feedback

- indirect or single feedback for coupled system

- memory type feedbacks

Generalizations to Nonlinear Feedbacks Assume now that the feedback term $a(x) u_{t}$ in (33) is replaced by a nonlinear feedback $a(x) \rho\left(u_{t}\right)$ where $\rho$ is a smooth, increasing function satisfying $v \rho(v) \geq 0$ for $v \in \mathbb{R}$, linear at $\infty$ and with polynomial growth close to zero, that is: $\rho(v)=|v|^{p}$ for $|v| \leq 1$ where $p \in(1, \infty)$.

Assume moreover that $\omega$ satisfies Zuazua's multiplier geometric condition (MGC) or Liu's piecewise multiplier method (PWMGC). Then using multipliers of the space and time variables defined as $E(s)^{(p-1) / 2} M u(x)$ where $M u(x)$ are multipliers of the form described in section 5.1 and integrating by parts expressions of the form

$$
\begin{aligned}
& \int_{t}^{T} E(s)^{(p-1) / 2} \\
& \quad \times \int_{\Omega}\left(\partial_{t}^{2} u-\Delta u+a(x) \rho\left(u_{t}\right)\right) M u(x) \mathrm{d} x \mathrm{~d} s=0,
\end{aligned}
$$

one can prove that the energy $E$ of solutions satisfies the following inequality for all $0 \leq t \leq T$

$$
\begin{aligned}
& \int_{t}^{T} E^{(p+1) / 2}(s) \mathrm{d} t \\
& \leq c E^{(p+1) / 2}(t)+c \int_{t}^{T} E^{(p-1) / 2}(s) \\
& \\
& \qquad \quad \times\left(\int_{\Omega} \rho\left(u_{t}\right)^{2}+\int_{\omega}\left|u_{t}\right|^{2}\right) .
\end{aligned}
$$

One can remark than an additional multiplicative weight in time depending on the energy has to be taken. This weight is $E^{(p-1) / 2}$. Then as in the linear case, but in a more involved way, thanks to the dissipation relation

$$
E^{\prime}(t)=-\int_{\Omega} a(x) u_{t} \rho\left(u_{t}\right),
$$

one can prove that $E$ satisfies the following nonlinear integral inequality

$$
\int_{t}^{T} E^{(p+1) / 2}(s) \mathrm{d} s \leq c E(t), \quad \forall 0 \leq t \leq T .
$$

Thanks to the fact that $E$ is nonincreasing, a wellknown result Komornik [74] shows that $E$ is polynomially decaying, as $t^{-2 /(p-1)}$ at infinity. The above type results have been obtained by many authors under weaker form (see also $[40,41,71,98,122]$ ).

Extensions to nonlinear feedbacks without growth conditions close to zero have been studied by Lasiecka and Tataru [78], Martinez [93], W. Liu and Zuazua [89], Eller Lagnese and Nicaise [56] and Alabau-Boussouira [5]. We present the results obtained in this last reference since they provide optimal decay rates.

The method is as follows. Define respectively the linear and nonlinear kinetic energies

$$
\left\{\begin{array}{l}
\int_{\omega}\left|u_{t}\right|^{2} \mathrm{~d} x, \\
\int_{\Omega}\left|a(x) \rho\left(u_{t}\right)\right|^{2} \mathrm{~d} x,
\end{array}\right.
$$

and use a weight function in time $f(E(s))$ which is to be determined later on in an optimal way. Integrating by parts 
${ }_{881} \int_{t}^{T} f(E(s)) \int_{\Omega}\left(\partial_{t}^{2} u-\Delta u+a(x) \rho\left(u_{t}\right)\right) M u(x) \mathrm{d} x \mathrm{~d} s=0$,

one can prove that the energy $E$ of solutions satisfies the following inequality for all $0 \leq t \leq T$

$$
\begin{array}{r}
\int_{t}^{T} E(s) f(E(s)) \mathrm{d} s \leq c f(E(t))+c \int_{t}^{T} f(E(s)) \\
\times\left(\int_{\Omega}\left|a(x) \rho\left(u_{t}\right)\right|^{2}+\int_{\omega}\left|u_{t}\right|^{2}\right) .
\end{array}
$$

The difficulty is to determine the optimal weight under general growth conditions on the feedback close to 0 , in particular for cases for which the feedback decays to 0 faster than polynomials.

Assume now that the feedback satisfies

$$
g(|v|) \leq|\rho(v)| \leq C g^{-1}(|v|), \quad \forall|v| \leq 1,
$$

where $g$ is continuously differentiable on $\mathbb{R}$ strictly increasing with $g(0)=0$ and

$$
\left\{\begin{array}{l}
g \in C^{2}\left(\left[0, r_{0}\right]\right), r_{0} \text { sufficiently small }, \\
H(.)=\sqrt{\cdot} \cdot g(\sqrt{\cdot}) \text { is strictly convex on }\left[0, r_{0}^{2}\right], \\
g \text { is odd }
\end{array}\right.
$$

Moreover, $\rho$ is assumed to have a linear growth with respect to the second variable at infinity. We define the optimal weight function $f$ as follows.

We first extend $H$ to a function $\hat{H}$ define on all $\mathbb{R}$

$$
\hat{H}(x)=\left\{\begin{array}{l}
H(x) \text { if } x \in\left[0, r_{0}^{2}\right], \\
+\infty \text { otherwise }
\end{array}\right.
$$

then, define a function $F$ as follows:

$$
F(y)= \begin{cases}\frac{\hat{H}^{*}(y)}{y} & \text { if } y \in(0,+\infty), \\ 0 & \text { if } y=0\end{cases}
$$

where $\hat{H}^{*}$ stands for the convex conjugate of $\hat{H}$, that is

$$
\hat{H}^{*}(y)=\sup _{x \in \mathbb{R}}\{x y-\hat{H}(x)\} .
$$

Then the optimal weight function $f$ is determined in the following way

$$
f(s)=F^{-1}(s / 2 \beta) \quad s \in\left[0,2 \beta r_{0}^{2}\right),
$$

where $\beta$ is of the form $\max \left(\eta_{1}, \eta_{2} E(0)\right), \eta_{1}$ and $\eta_{2}$ being explicit positive constants.
One can prove that the above formulas make sense, and in particular that $F$ is invertible and smooth. More precisely, $F$ is twice continuously differentiable strictly increasing, one-to-one function from $[0,+\infty)$ onto $\left[0, r_{0}^{2}\right)$. Note that since the feedback is supposed to be linear at infinity, if one wants to obtain results for general growth types of the feedback, one can assume convexity of $H$ only in a neighborhood of 0 .

One can prove from (41) that there exists an (explicit) $T_{0}>0$ such that for all initial data, $E$ satisfies the following nonlinear integral inequality

$$
\int_{t}^{T} E(s) f(E(s)) \mathrm{d} s \leq T_{0} E(t) \quad \forall 0 \leq t \leq T .
$$

This inequality is proved thanks to convexity arguments as follows. Thanks to the convexity of $\hat{H}$, one can use Jensen's inequality and 42 ), so that

$$
\begin{aligned}
\int_{\Omega_{t}}\left|a(x) \rho\left(u_{t}\right)\right|^{2} \mathrm{~d} x & \leq \gamma_{1}(t) \hat{H}^{-1} \\
& \times\left(\frac{1}{\gamma_{1}(t)} \int_{\Omega} a(x) u_{t} \rho\left(u_{t}\right) \mathrm{d} x\right)
\end{aligned}
$$

911 912 913 914 915 916 917

In a similar way, one proves that

$$
\int_{\omega_{t}}\left|u_{t}\right|^{2} \mathrm{~d} x \leq \gamma_{2}(t) \hat{H}^{-1}\left(\frac{1}{\gamma_{2}(t)} \int_{\Omega} a(x) u_{t} \rho\left(u_{t}\right) \mathrm{d} x\right) \quad{ }_{931}
$$

where $\Omega_{t}$ and $\omega_{t}$ are time-dependent sets of respective Lebesgue measures $\gamma_{1}(t)$ and $\gamma_{2}(t)$ on which the velocity $u_{t}(t, x)$ is sufficiently small. Using the above two estimates, together with the linear growth of $\rho$ at infinity, one proves

$$
\begin{aligned}
\int_{t}^{T} f(E(s))\left(\int_{\Omega}\left|a(x) \rho\left(u_{t}\right)\right|^{2}+\int_{\omega}\left|u_{t}\right|^{2}\right) \\
\leq \int_{t}^{T} f(E(s)) \hat{H}^{-1}\left(\frac{1}{c} \int_{\Omega} a(x) u_{t} \rho\left(u_{t}\right) \mathrm{d} x\right)
\end{aligned}
$$

Using then Young's inequality, together with the dissipation relation (40) in the above inequality, one obtains

$$
\begin{array}{r}
\int_{t}^{T} f(E(s))\left(\int_{\Omega}\left|a(x) \rho\left(u_{t}\right)\right|^{2}+\int_{\omega}\left|u_{t}\right|^{2}\right) \\
\leq C_{1} \int_{t}^{T} \hat{H}^{\star}\left(f(E(s)) \mathrm{d} s+C_{2} E(t),\right.
\end{array}
$$

where $C_{i}>0 i=1,2$ is a constant independent of the ini- ${ }_{943}$ tial data. Using the dissipation relation (40) in the above 944 inequality, this gives for all $0 \leq t \leq T$

Combining this last inequality with (41) give

$\int_{t}^{T} E(s) f(E(s)) \mathrm{d} s \leq \beta \int_{t}^{T}(\hat{H})^{\star}\left(f(E(s)) \mathrm{d} s+C_{2} E(t)\right.$ 
where $\beta$ is chosen of the form $\max \left(\eta_{1}, \eta_{2} E(0)\right), \eta_{1}$ and $\eta_{2}$ being explicit positive constants to guarantee that the argument $E$ of $f$ stays in the domain of definition of $f$. Thus (43) is proved, thanks to the fact that the weight function has been chosen so that

$$
\beta \hat{H}^{\star}\left(f(E(s))=\frac{1}{2} E(s) f(E(s)) \quad \forall 0 \leq s .\right.
$$

Therefore $E$ satisfies a nonlinear integral inequality with a weight function $f(E)$ which is defined in a semi-explicit way in general cases of feedback growths.

The last step is to prove that a nonincreasing and nonnegative absolutely continuous function $E$ satisfying a nonlinear integral inequality of the form (43) is decaying at infinity, and to establish at which rate this holds. For this, one proceeds as in [5].

Let $\eta>0$ and $T_{0}>0$ be fixed given real numbers and $F$ be a strictly increasing function from $[0,+\infty)$ on $[0, \eta)$, with $F(0)=0$ and $\lim y \rightarrow+\infty F(y)=\eta$.

For any $r \in(0, \eta)$, we define a function $K_{r}$ from $(0, r]$ on $[0,+\infty)$ by

$$
K_{r}(\tau)=\int_{\tau}^{r} \frac{\mathrm{d} y}{y F^{-1}(y)},
$$

and a function $\psi_{r}$ which is a strictly increasing onto function defined from $\left[\frac{1}{F^{-1}(r)},+\infty\right)$ on $\left[\frac{1}{F^{-1}(r)},+\infty\right)$ by

$$
\psi_{r}(z)=z+K_{r}\left(F\left(\frac{1}{z}\right)\right) \geq z, \quad \forall z \geq \frac{1}{F^{-1}(r)},
$$

Then one can prove that if $E$ is a nonincreasing, absolutely continuous function from $[0,+\infty)$ on $[0,+\infty)$, satisfying $0<E(0)<\eta$ and the inequality

$$
\int_{t}^{T} E(s) F^{-1}(E(s)) \mathrm{d} s \leq T_{0} E(S), \quad \forall 0 \leq t \leq T .
$$

Then $E$ satisfies the following estimate:

$$
E(t) \leq F\left(\frac{1}{\psi_{r}^{-1}\left(\frac{t}{T_{0}}\right)}\right), \quad \forall t \geq \frac{T_{0}}{F^{-1}(r)},
$$

where $r$ is any real such that

$$
\frac{1}{T_{0}} \int_{0}^{+\infty} E(\tau) F^{-1}(E(\tau)) \mathrm{d} \tau \leq r \leq \eta .
$$

Thus, one can apply the above result to $E$ with $\eta=r_{0}^{2}$ and show that $\lim t \rightarrow+\infty E(t)=0$, the decay rate being given by the estimate (48).

If $g$ is polynomial close to zero, one gets back that the energy $E(t)$ decays as $t^{\frac{-2}{p-1}}$ at infinity. If $g(v)$ behaves as $\exp (-1 /|v|)$ close to zero, then $E(t)$ decays as $1 /(\ln (t))^{2}$ at $\quad{ }_{984}$ infinity.

The usefulness of convexity arguments has been first pointed out by Lasiecka and Tataru [78] using Jensen's inequality and then in different ways by Martinez [93] (the weight function does not depend on the energy) and W. Liu and Zuazua [89] and Eller Lagnese and Nicaise [56]. Optimal decay rates have been obtained by Alabau-Boussouira [5,6] using a weight function determined through the theory of convex conjugate functions and Young's (named also as Fenchel-Moreau's) inequality. This argument was also used by W. Liu and Zuazua [89] in a slightly different way and combined to a Lyapunov technique. Optimality of estimates in [5] is proved in one-dimensional situation and for boundary dampings applying optimality results of Vancostenoble [119] (see also Martinez and Vancostenoble [118]).

\section{Indirect Damping for Coupled Systems}

Many complex phenomena are modelized through coupled systems. In stabilizing (or controlling) energies of the vector state, one has very often access only to some components of this vector either due to physical constraints or to cost considerations. In this case, the situation is to stabilize a full system of coupled equation through a reduced number of feedbacks. This is called indirect damping. This notion has been introduced by Russell [109] in 1993.

As an example, we consider the following system:

$$
\left\{\begin{array}{l}
\partial_{t}^{2} u-\Delta u+\partial_{t} u+\alpha v=0 \\
\partial_{t}^{2} v-\Delta v+\alpha u=0 \\
\quad \text { in } \Omega \times \mathbb{R}, \quad u=0=v \quad \text { on } \partial \Omega \times \mathbb{R} .
\end{array}\right.
$$

Here, the first equation is damped through a linear distributed feedback, while no feedback is applied to the second equation. The question is to determine if this coupled system inherits any kind of stability for nonzero values of the coupling parameter $\alpha$ from the stabilization of the first equation only.

In the finite dimensional case, stabilization (or control) of coupled ODE's can be analyzed thanks to a powerful rank type condition named Kalman's condition. The situation is much more involved in the case of coupled PDE's.

One can show first show that the above system fails to be exponentially stable (see also [66] for related results). More generally, one can study the stability of the system

$$
\left\{\begin{array}{l}
u^{\prime \prime}+A_{1} u+B u^{\prime}+\alpha v=0 \\
v^{\prime \prime}+A_{2} v+\alpha u=0
\end{array}\right.
$$


in a separable Hilbert space $H$ with norm $|\cdot|$, where $A_{1}, A_{2}$ and $B$ are self-adjoint positive linear operators in $H$. Moreover, $B$ is assumed to be a bounded operator. So, our analysis applies to systems with internal damping supported in the whole domain $\Omega$ such as (49); the reader is referred to $[1,2]$ for related results concerning boundary stabilization problems (see also Beyrath $[23,24]$ for localized indirect dampings).

In light of the above observations, system (50) fails to be exponentially stable, at least when $H$ is infinite dimensional and $A_{1}$ has a compact resolvent as in (49). Indeed it is shown in Alabau, Cannarsa and Komornik [8] that the total energy of sufficiently smooth solutions of (50) decays polynomially at infinity whenever $|\alpha|$ is small enough but nonzero. From this result we can also deduce that any solution of (50) is strongly stable regardless of its smoothness: this fact follows by a standard density argument since the semigroup associated with (50) is a contraction semigroup.

A brief description of the key ideas of the approach developed in $[2,8]$ is as follows. Essentially, one uses a finite iteration scheme and suitable multipliers to obtain an estimate of the form

$$
\int_{0}^{T} E(u(t), v(t)) \mathrm{d} t \leq c \sum_{k=0}^{j} E\left(u^{(k)}(0), v^{(k)}(0)\right)
$$$$
\forall T \geq 0,
$$

where $j$ is a positive integer and $E$ denotes the total energy of the system

$$
\begin{aligned}
E(u, v)=\frac{1}{2}\left(\left|A_{1}^{1 / 2} u\right|^{2}+\left|u^{\prime}\right|^{2}\right) & \\
& +\frac{1}{2}\left(\left|A_{2}^{1 / 2} v\right|^{2}+\left|v^{\prime}\right|^{2}\right)+\alpha\langle u, v\rangle .
\end{aligned}
$$

Once (51) is proved, an abstract lemma due to Alabau [1,2] shows that $E(u(t), v(t))$ decays polynomially at $\infty$. This abstract lemma can be stated as follows.

Let $A$ be the infinitesimal generator of a continuous semi-group $\exp (t A)$ on an Hilbert space $\mathcal{H}$, and $D(A)$ its domain. For $U^{0}$ in $\mathcal{H}$ we set in all the sequel $U(t)=$ $\exp (t A) U^{0}$ and assume that there exists a functional E defined on $C([0,+\infty), \mathcal{H})$ such that for every $U^{0}$ in $\mathcal{H}$, $E(\exp (. A))$ is a non-increasing, locally absolutely continuous function from $[0,+\infty)$ on $[0,+\infty)$. Assume moreover that there exist an integer $k \in \mathbb{N}^{\star}$ and nonnegative constants $c_{p}$ for $p=0, \ldots k$ such that

$$
\begin{aligned}
\int_{S}^{T} E(U(t)) \mathrm{d} t \leq & \sum_{p=0}^{k} c_{p} E\left(U^{(p)}(S)\right) \\
& \forall 0 \leq S \leq T, \forall U^{0} \in D\left(A^{k}\right) .
\end{aligned}
$$

Then the following inequalities hold for every $U^{0}$ in 1078 $D\left(A^{k n}\right)$ and all $0 \leq S \leq T$ where $n$ is any positive integer:

$$
\int_{S}^{T} E(U(\tau)) \frac{(\tau-S)^{n-1}}{(n-1) !} \mathrm{d} \tau \leq c \sum_{p=0}^{k n} E\left(U^{(p)}(S)\right),
$$

and

$$
\begin{aligned}
& E(U(t)) \leq c \sum_{p=0}^{k n} E\left(U^{(p)}(0)\right) t^{-n} \\
& \forall t>0, \quad \forall U^{0} \in D\left(A^{k n}\right),
\end{aligned}
$$

where $c$ is a constant which depends on $n$.

First (53) is proved by induction on $n$. For $n=1$, it reduces to the hypothesis (52). Assume now that (53) holds for $n$ and let $U^{0}$ be given in $D\left(A^{k(n+1)}\right)$. Then we have

$$
\begin{aligned}
& \int_{S}^{T} \int_{t}^{T} E(U(\tau)) \frac{(\tau-t)^{n-1}}{(n-1) !} \mathrm{d} \tau \mathrm{d} t \\
& \leq c \sum_{p=0}^{k n} \int_{S}^{T} E\left(U^{(p)}(t)\right) \mathrm{d} t \\
& \forall 0 \leq S \leq T, \forall U^{0} \in D\left(A^{k n}\right) .
\end{aligned}
$$

Since $U^{0}$ is in $D\left(A^{k(n+1)}\right)$ we deduce that $U^{(p)}(0)=A^{p} U^{0} \quad 1095$ is in $D\left(A^{k}\right)$ for $p \in\{0, \ldots k n\}$. Hence we can apply the assumption (52) to the initial data $U^{(p)}(0)$. This together with Fubini's Theorem applied on the left hand side of the above inequality give (53) for $n+1$. Using the property that $E(U(t))$ is non increasing in (53) we easily obtain the last desired inequality.

Applications on wave-wave, wave-Petrowsky equations and various concrete examples hold.

The above results have been studied later on by Batkai, Engel, Prüss and Schnaubelt [18] using very interesting resolvent and spectral criteria for polynomial stability of abstract semigroups. The above abstract lemma in [2] has also been generalized using interpolation theory. One should note that this integral inequality involving higher order energies of solutions is not of differential nature contrarily to the Haraux's and Komornik's integral inequalities. Another approach based on decoupling techniques

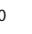
.

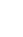

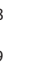

1090 
and for slightly different abstract systems have been introduced by Ammar Khodja Bader and Ben Abdallah [12].

Spectral conditions have also been studied by $\mathrm{Z}$. Liu [88] and later on by Z. Liu and Rao [90], Loreti and Rao [92] for peculiar abstract systems and in general for coupled equations only of the same nature (wave-wave for instance), so that a dispersion relation for the eigenvalues of the coupled system can be derived. Also these last results are given for internal stabilization only. From the above limitations, Z. Liu-Rao and Loreti-Rao's results are less powerful in generality than the ones given by Alabau, Cannarsa and Komornik [8] and Alabau [2]. Moreover results through energy type estimates and integral inequalities can be generalized to include nonlinear indirect dampings as shown in [7]. On the other side spectral methods are very precise for the obtention of optimal decay rates provided that one can determine at which speed the eigenvalues approach the imaginary axis for high frequencies.

\section{Memory Dampings}

We consider the following model problem

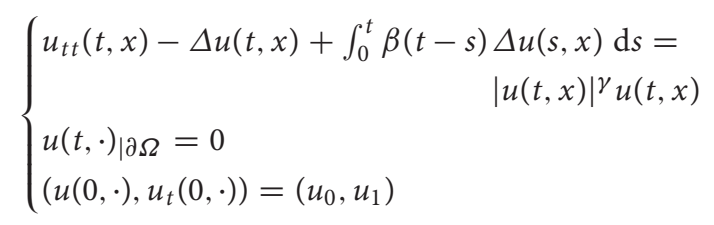

where $0<\gamma \leq \frac{2}{N-2}$ holds. The second member is a source term. The damping

$$
\int_{0}^{t} \beta(t-s) \Delta u(s, x) \mathrm{d} s
$$

is of memory type.

The energy is defined by

$$
\begin{aligned}
E_{u}(t)= & \frac{1}{2}\left\|u_{t}(t)\right\|_{L^{2}(\Omega)}^{2} \mathrm{~d} x \\
& +\frac{1}{2}\left(1-\int_{0}^{t} \beta(s) \mathrm{d} s\right)\|\nabla u(t)\|_{L^{2}(\Omega)}^{2} \\
& -\frac{1}{\gamma+2}\|u(t)\|_{L^{\gamma+2}(\Omega)}^{\gamma+2} \\
& +\frac{1}{2} \int_{0}^{t} \beta(t-s)\|\nabla u(t)-\nabla u(s)\|_{L^{2}(\Omega)}^{2} \mathrm{~d} s
\end{aligned}
$$

The damping term produces dissipation of the energy, that $\quad 1140$ is (for strong solutions)

$$
\begin{aligned}
E_{u}^{\prime}(t)=-\frac{1}{2} \beta(t)\|\nabla u(t)\|^{2} & \\
& +\frac{1}{2} \int_{0}^{t} \beta^{\prime}(t)\|\nabla u(s)-\nabla u(t)\|^{2} \mathrm{~d} s \leq 0
\end{aligned}
$$

One can consider more general abstract equations of the form

$$
\begin{aligned}
u^{\prime \prime}(t)+A u(t)-\int_{0}^{t} \beta(t-s) A u(s) \mathrm{d} s & =\nabla F(u(t)) \\
t & \in(0, \infty)
\end{aligned}
$$

in a Hilbert space $X$, where $A: D(A) \subset X \rightarrow X$ is an accretive self-adjoint linear operator with dense domain, and $\nabla F$ denotes the gradient of a Gâteaux differentiable functional $F: D\left(A^{1 / 2}\right) \rightarrow \mathbb{R}$. In particular, equation (54) fits into this framework as well as several other classical equations of mathematical physics such as the linear elasticity system.

We consider the following assumptions.

\section{Assumptions (H1)}

1. $A$ is a self-adjoint linear operator on $X$ with dense domain $D(A)$, satisfying

$$
\langle A x, x\rangle \geq M\|x\|^{2} \quad \forall x \in D(A)
$$

for some $M>0$.

2. $\beta:[0, \infty) \rightarrow[0, \infty)$ is a locally absolutely continuous function such that

$$
\int_{0}^{\infty} \beta(t) \mathrm{d} t<1 \beta(0)>0 \quad \beta^{\prime}(t) \leq 0 \quad 1168
$$

for a.e. $t \geq 0 . \quad 1160$

3. $F: D\left(A^{1 / 2}\right) \rightarrow \mathbb{R}$ is a functional such that

1. $F$ is Gâteaux differentiable at any point $x \in D\left(A^{1 / 2}\right) ; \quad 1172$

2. for any $x \in D\left(A^{1 / 2}\right)$ there exists a constant $c(x)>0 \quad 1173$ such that

$$
|D F(x)(y)| \leq c(x)\|y\|, \quad \text { for any } y \in D\left(A^{1 / 2}\right), \quad 1175
$$

where $D F(x)$ denotes the Gâteaux derivative of $F$ in $\quad 1176$ $x$; consequently, $D F(x)$ can be extended to the whole space $X$ (and we will denote by $\nabla F(x)$ the unique vector representing $D F(x)$ in the Riesz isomorphism, that is, $\langle\nabla F(x), y\rangle=D F(x)(y)$, for any $y \in X)$;
1152 1153 1154 1155 1156 1157 
3. for any $R>0$ there exists a constant $C_{R}>0$ such that

$$
\|\nabla F(x)-\nabla F(y)\| \leq C_{R}\left\|A^{1 / 2} x-A^{1 / 2} y\right\|
$$

for all $x, y \in D\left(A^{1 / 2}\right)$ satisfying $\left\|A^{1 / 2} x\right\|,\left\|A^{1 / 2} y\right\| \leq$ $R$.

\section{Assumptions (H2)}

1. There exist $p \in(2, \infty]$ and $k>0$ such that

$$
\beta^{\prime}(t) \leq-k \beta^{1+\frac{1}{p}}(t) \text { for a.e. } t \geq 0
$$

(here we have set $\frac{1}{p}=0$ for $p=\infty$ ).

2. $F(0)=0, \nabla F(0)=0$, and there is a strictly increasing continuous function $\psi:[0, \infty) \rightarrow[0, \infty)$ such that $\psi(0)=0$ and

$$
|\langle\nabla F(x), x\rangle| \leq \psi\left(\left\|A^{1 / 2} x\right\|\right)\left\|A^{1 / 2} x\right\|^{2} \quad \forall x \in D\left(A^{1 / 2}\right) .
$$

Under these assumptions, global existence for sufficiently small (resp. all) initial data in the energy space can be proved for nonvanishing (resp. vanishing) source terms.

It turns out that the above energy methods based on multiplier techniques combined with linear and nonlinear integral inequalities can be extended to handle memory dampings and applied to various concrete examples such as wave, linear elastodynamic and Petrowsky equations for instance. This allows to show in [10] that exponential as well as polynomial decay of the energy hold if the kernel decays respectively exponentially or polynomially at infinity.

The method is as follows. One evaluates expressions of the form

$$
\int_{t}^{T}\left\langle u^{\prime \prime}(s)+A u(s)-\int_{0}^{t} \beta * A u(s)-\nabla F(u(s), M u\rangle \mathrm{d} s\right.
$$

where the multipliers $M u$ are of the form $\phi(s)\left(c_{1}(\beta *\right.$ $\left.u)(s)+c_{2}(s) u\right)$ with $\phi$ which is a differentiable, nonincreasing and nonnegative function, and $c_{1}$ being a suitable constant, whereas $c_{2}$ may be chosen dependent on $\beta$.

Integrating by parts the resulting relations and performing some involved estimates, one can prove that for all $t_{0}>0$ and all $T \geq t \geq t_{0}$

$$
\begin{aligned}
& \int_{t}^{T} \phi(s) E(s) \mathrm{d} s \leq C \phi(0) E(t)+\int_{t}^{T} \phi(s) \\
& \quad \times \int_{0}^{s} \beta(s-\tau)\left\|A^{1 / 2} u(s)-A^{1 / 2} u(\tau)\right\|^{2} \mathrm{~d} \tau \mathrm{d} s,
\end{aligned}
$$

If $p=\infty$, that is if the kernel $\beta$ decays exponentially, one can easily bound the last term of the above estimate by $c E(t)$ thanks to the dissipation relation.

If $p \in(2, \infty)$, one has to proceed differently since the term

$$
\int_{t}^{T} \phi(s) \int_{0}^{s} \beta(s-\tau)\left\|A^{1 / 2} u(s)-A^{1 / 2} u(\tau)\right\|^{2} d \tau \mathrm{d} s
$$

1221 1222

cannot be directly estimated thanks to the dissipation relation. To bound this last term, one can generalize an argument of Cavalcanti and Oquendo [37] as follows. Define, for any $m \geq 1$,

$$
\begin{array}{r}
\varphi_{m}(t):=\int_{0}^{t} \beta^{1-\frac{1}{m}}(t-s)\left\|A^{1 / 2} u(s)-A^{1 / 2} u(t)\right\|^{2} \mathrm{~d} s \\
t \geq 0
\end{array}
$$

Then, we have for any $T \geq S \geq 0$

$$
\begin{gathered}
\int_{S}^{T} E_{u}^{\frac{m}{p}}(t) \int_{0}^{t} \beta(t-s)\left\|A^{1 / 2} u(s)-A^{1 / 2} u(t)\right\|^{2} \mathrm{~d} s \mathrm{~d} t \\
\leq C E_{u}^{\frac{p}{p+m}}(S)\left(\int_{S}^{T} E_{u}^{1+\frac{m}{p}}(t) \varphi_{m}(t) \mathrm{d} t\right)^{\frac{m}{p+m}}
\end{gathered}
$$

for some constant $C>0$. Then one proves Suppose that, if for some $m \geq 1$, the function $\varphi_{m}$ defined in (57) is bounded. Then, for any $S_{0}>0$ there is a positive constant $C$ such that

$$
\begin{array}{r}
\int_{S}^{\infty} E_{u}^{1+\frac{m}{p}}(t) \mathrm{d} t \leq C\left(E_{u}^{\frac{m}{p}}(0)+\left\|\varphi_{m}\right\|_{\infty}^{\frac{m}{p}}\right) E_{u}(S) \\
\forall S \geq S_{0} .
\end{array}
$$

One uses this last result first with $m=2$ noticing that $\varphi_{2}$ is ${ }_{1248}$ bounded and $\phi=E^{2 / p}$. This gives a first energy decay rate as $(t+1)^{-p / 2}$. This estimate shows that $\varphi_{1}$ is bounded. Then one applies once again the last result with $m=1$ and $\phi=E^{1 / p}$. One deduces then that $E$ decays as $(t+1)^{-p}$ which is the optimal decay rate expected.

\section{Bibliographical Comments}

For an introduction to the multiplier method, we refer the interested reader to the books of J.-L. Lions [86], Komornik [74] and the references therein. The celebrated result of Bardos Lebeau and Rauch is presented in [86]. A general abstract presentation of control problems for hyperbolic and parabolic equations can be found in the book of Lasiecka and Triggiani [80,81]. Results on spectral methods and the frequency domain approach can be 
found in the book of Z. Liu [88]. There also exists an interesting approach developed for bounded feedback operators by Haraux and extended to the case of unbounded feedbacks by Ammari and Tucsnak [11]. In this approach, the polynomial (or exponential) stability of the damped system is proved thanks to the corresponding observability for the undamped (conservative) system. Such observability results for weakly coupled undamped systems have been obtained for instance in [3].

Many other very interesting issues have been studied connected to semilinear wave equations $[34,123]$ and the references therein, to the case of wave damped equations with nonlinear source terms [39].

Well-posedness and asymptotic properties for PDE's with memory terms have first been studied by Dafermos [53,54] for convolution kernels with past history (convolution up to $t=-\infty$ ), by Prüss [103] and Prüss and Propst [102] in which the efficiency of different models of dampings are compared to experiments (see also Londen Petzeltova and Prüss [91]). Decay estimates for the energy of solutions using multiplier methods combined with Lyapunov type estimates for an equivalent energy are proved in Munoz Rivera [97], Munoz Rivera and Salvatierra [96], Cavalcanti and Oquendo [37] and Giorgi Naso and Pata [67] and many other papers.

\section{Optimal Control}

As for positional control, also for optimal control problems it is convenient to adopt the abstract formulation introduced in Sect. "Abstract Evolution Equations". Let the state space be represented by the Hilbert space $H$, and the state equation be given in the form (12), that is

$$
\left\{\begin{array}{l}
u^{\prime}(t)=A u(t)+B f(t) \quad t \in[0, T] \\
u(0)=u_{0}
\end{array}\right.
$$

Recall that $A$ is the infinitesimal of a strongly continuous semigroup, $e^{t A}$, in $H, B$ is a (bounded) linear operator from $F$ (the control space) to $H$, and $u_{f}$ stands for the unique (mild) solution of (60) for a given control function $f \in L^{2}(0, T ; H)$.

A typical optimal control problem of interest for PDE's is the Bolza problem which consists in

$$
1302 \quad\left\{\begin{array}{l}
\text { minimizing the cost functional } \\
J(f) \doteq \int_{0}^{T} L\left(t, u_{f}(t), f(t)\right) \mathrm{d} t+\ell\left(u_{f}(T)\right) \\
\text { over all controls } f \in L^{2}(0, T ; F) .
\end{array}\right.
$$

Here, $T$ is a positive number, called the horizon, whereas $L$ and $\ell$ are given functions, called the running cost and final cost, respectively. Such functions are usually assumed to be bounded below.

A control function $f_{*} \in L^{2}(0, T ; F)$ at which the above minimum is attained is called an optimal control for problem (61) and the corresponding solution $u_{f_{*}}$ of (60) is said to be an optimal trajectory. Alltogether, $\left\{u_{f_{*}}, f_{*}\right\}$ is called an optimal (trajectory/control) pair.

For problem (61) the following issues will be addressed in the sections below:

- the existence of controls minimizing functional $J$;

- necessary conditions that a candidate solution must satisfy;

- sufficient conditions for optimality provided by the dynamic programming method.

Other problems of particular interest to CT for PDE's are problems with an infinite horizon $(T=\infty)$, problems with a free horizon $T$ and a final target, and problems with constraints on both control variables and state variables. Moreover, the study of nonlinear variants of (60), including semilinear problems of the form

$$
\left\{\begin{array}{l}
u^{\prime}(t)=A u(t)+h(t, u(t), f(t)) \quad t \in[0, T] \\
u(0)=u_{0}
\end{array}\right.
$$

is strongly motivated by applications. The discussion of all these variants, however, will not be here pursued in detail.

Traditionally, in optimal control theory, state variables are denoted by the letters $x, y, \ldots$, whereas $u, v, \ldots$ are reserved for control variables. For notational consistency, in this section $u(\cdot)$ will still denote the state of a given system and $f(\cdot)$ a control function, while $\phi$ will stand for a fixed element of control space $F$.

\section{Existence of Optimal Controls}

From the study of finite dimensional optimization it is a familiar fact that the two essential ingredients to guarantee the existence of minima are compactness and lower semicontinuity. Therefore, it is clear that, in order to obtain a solution of the optimal control problem (60)-(61), one has to make assumptions that allow to recover such properties. The typical hypotheses that are made for this purpose are the following:

- coercivity: there exist constants $c_{0}>0$ and $c_{1} \in \mathbb{R}$ such that

$$
\begin{aligned}
& \ell(\phi) \geq c_{1} \quad \text { and } L(t, u, \phi) \geq c_{0}\|\phi\|^{2}+c_{1} \\
& \forall(t, u, \phi) \in[0, T] \times H \times F
\end{aligned}
$$


- convexity: for every $(t, u) \in[0, T] \times H$

$$
\phi \mapsto L(t, u, \phi) \quad \text { is convex on } \quad F
$$

Under the above hypotheses, assuming lower semicontinuity of $\ell$ and of the map $L(t, \cdot, \phi)$, it is not hard to show that problem (60)-(61) has at least one solution. Indeed, assumption (63) allows to show that any minimizing sequence of controls $\left\{f_{k}\right\}$ is bounded in $L^{2}(0, T ; H)$. So, it admits a subsequence, still denoted by $\left\{f_{k}\right\}$ which converges weakly in $L^{2}(0, T ; H)$ to some function $f$. Then, by linearity, $u_{f_{k}}(t)$ converges to $u_{f}(t)$ for every $t \in[0, T]$. So, using assumption (64), it follows that $f$ is a solution of (60)-(61).

The problem becomes more delicate when the Tonelli type coercivity condition (63) is relaxed, or the state equation is nonlinear as in (62). Indeed, the convergence of $u_{f_{k}}(t)$ is no longer ensured, in general. So, in order to recover compactness, one has to make further assumptions, such as the compactness of $e^{t A}$, or structural properties of $L$ and $h$. For further reading, one may consult the monographs [22,85], and [79], for problems where the running and final costs are given by quadratic forms (the so-called Linear Quadratic problem), or [84] and [59] for more general optimal control problems.

\section{Necessary Conditions}

Once the existence of a solution to problem (60)-(61) has been established, the next important step is to provide conditions to detect a candidate solution, possibly showing that it is, in fact, optimal. By and large the optimality conditions of most common use are the ones known as Pontryagin's Maximum Principle, named after the Russian mathematician L.S. Pontryagin who greatly contributed to the development of control theory, see $[100,101]$.

So, suppose $\left\{u_{*}, f_{*}\right\}$, where $u_{*}=u_{f_{*}}$ is a candidate optimal pair, and consider the so-called adjoint system

$\left\{\begin{array}{l}-p^{\prime}(t)=A^{*} p(t)+\partial_{u} L\left(t, u_{*}(t), f_{*}(t)\right)=0 \\ \quad t \in[0, T] \text { a.e. } \\ p(T)=\partial \ell\left(u_{*}(T)\right),\end{array}\right.$

where $\partial_{u} L(t, u, \phi)$ and $\partial \ell(u)$ denote the Fréchet gradients of the maps $L(t, \cdot, \phi)$ and $\ell$ at $u$, respectively. Observe that the above is a backward linear Cauchy problem with terminal condition, which can obviously be reduced to a forward one by the change of variable $t \rightarrow T-t$. So, it admits a unique mild solution, labeled $p_{*}$, which is called the adjoint state associated with $\left\{u_{*}, f_{*}\right\}$.
Pontryagin's Maximum Principle states that, if $\left\{u_{*}, \quad 1391\right.$ $\left.f_{*}\right\}$ is optimal, then

$$
\begin{array}{r}
\left\langle p_{*}(t), B f_{*}(t)\right\rangle+L\left(t, u_{*}(t), f_{*}(t)\right)= \\
\min _{\phi \in F}\left[\left\langle p_{*}(t), B \phi\right\rangle+L\left(t, u_{*}(t), \phi\right)\right]
\end{array}
$$$$
t \in[0, T] \text { a.e. }
$$

The name Maximum Principle rather than Minimum Principle, as it would be more appropriate, is due to the fact that, traditionally, attention was focussed on the maximization-instead of minimization-of the functional in (61). Even today, in most models from economics, one is interested in maximizing payoffs, such as revenues, utility, capital and so on. In that case, (65) would still be true, with a "max" instead of a "min".

At first glance, it might be hard to understand the revelance of (65) to problem (61). To explain this, introduce the function, called the Hamiltonian,

$$
\begin{aligned}
\mathcal{H}(t, u, p)=\min _{\phi \in F}[\langle p, B \phi\rangle+L(t, u, \phi)] & \\
& (t, u, p) \in[0, T] \times H \times H .
\end{aligned}
$$

Then, Fermat's rule yields $B^{*} p+\partial_{\phi} L(t, u, \phi)=0$ at every $\phi \in F$ at which the minimum in (66) is attained. Therefore, from (65) it follows that

$$
B^{*} p_{*}(t)+\partial_{\phi} L\left(t, u_{*}(t), f_{*}(t)\right)=0 \quad t \in[0, T] \text { a.e. (67) }
$$

which provides a much-easier-to-use optimality condition.

There is a vast literature on necessary condition for optimality for distributed parameter systems. The set-up that was considered above can be generalized in several ways: one can consider nonlinear state equations as in (62), nonsmooth running and finals costs, constraints on both state and control, problems with infinite horizon or exit times. Further reading and useful references on most of these extensions can be found in the aforementioned monographs $[22,79,84,85]$, and in [59] which is mainly concerned with time optimal control problems.

\section{Dynamic Programming}

Though useful as it may be, Pontryagin's Maximum Principle remains a necessary condition. So, without further information, it does not suffice to prove the optimality of a give trajectory/control pair. Moreover, even when the map $\phi \mapsto \partial_{\phi} L(t, u, \phi)$ turns out to be invertible, the best result identity $(67)$ can provide, is a representation of $f_{*}(t)$ in terms of $u_{*}(t)$ and $p_{*}(t)$ : not enough to determine $f_{*}(t)$, in general. 
This is why other methods to construct optimal controls have been proposed over the years. One of the most interesting ones is the so-called dynamic programming method (abbreviated, DP), initiated by the work of R. Bellman [20]. Such a method will be briefly described below in the set-up of distributed parameter systems.

Fix $T>0, s$ such that $0 \leq s \leq T$, and consider the optimal control problem

$$
\begin{aligned}
& \text { to minimize } \\
& J^{s, v}(f)=\int_{s}^{T} L\left(t, u_{f}^{s, v}(t), f(t)\right) \mathrm{d} t+\ell\left(u_{f}^{s, v}(T)\right)
\end{aligned}
$$

over all control functions $f \in L^{2}(s, T ; F)$, where $u_{f}^{s, v}(t)$ is the solution of the controlled system

$$
\left\{\begin{array}{l}
u^{\prime}(t)=A u(t)+B f(t) \quad t \in[s, T] \\
u(s)=v
\end{array}\right.
$$

The value function $U$ associated to (68)-(69) is the realvalued function defined by

$$
U(s, v)=\inf _{f \in L^{2}(s, T ; F)} J^{s, v}(f) \quad \forall(s, v) \in[0, T] \times H .
$$

A fundamental step of DP is the following result, known as Bellman's optimality principle.

Theorem 5 For any $(s, v) \in[0, T] \times H$ and any $f \in$ $L^{2}(s, T ; F)$

$$
U(s, v) \leq \int_{s}^{r} L\left(t, u_{f}^{s, v}(t), f(t)\right) \mathrm{d} t+U\left(r, u_{f}^{s, v}(r)\right)
$$$$
\forall r \in[s, T] .
$$

Moreover, $f^{*}(\cdot)$ is optimal if and only if

$$
\begin{array}{r}
U(s, v)=\int_{s}^{r} L\left(t, u_{f}^{s, v}(t), f(t)\right) \mathrm{d} t+U\left(r, u_{f}^{s, v}(r)\right) \\
\forall r \in[s, T] .
\end{array}
$$

The connection between DP and optimal control is based on the properties of the value function. Indeed, applying Bellman's optimality principle, one can show that, if $U$ is Fréchet differentiable, then

$$
\left\{\begin{array}{l}
\partial_{s} U(s, v)+\left\langle A v, \partial_{v} U(s, v)\right\rangle+\mathcal{H}\left(s, v, \partial_{v} U(s, v)\right)=0 \\
U(T, v)=\ell(v) \quad v \in H
\end{array}\right.
$$

where $\mathcal{H}$ is the Hamiltonian defined in (66). The above equation is the celebrated Hamilton-Jacobi equation of
DP. To illustrate its connections with the original optimal control problem, a useful formal argument-that can, however, be made rigorous-is the following. Consider a sufficiently smooth solution $W$ of the above problem and let $(s, v) \in(0, T) \times D(A)$. Then, for any trajectory/control pair $\{u, f\}$,

$$
\begin{aligned}
\frac{\mathrm{d}}{\mathrm{d} t} W(t, u(t))= & \partial_{s} W(t, u(t))+\left\langle\partial_{\nu} W(t, u(t)), A u(t)\right. \\
& +B f(t)\rangle \\
= & \left\langle\partial_{\nu} W(t, u(t)), B f(t)\right\rangle \\
& -\mathcal{H}\left(t, u(t), \partial_{\nu} W(t, u(t))\right) \\
\geq & -L(t, u(t), f(t))
\end{aligned}
$$

by the definition of $\mathcal{H}$. Therefore, integrating from $s$ to $T$,

$$
\ell(u(T))-W(s, v) \geq-\int_{s}^{T} L(t, u(t), f(t)) \mathrm{d} t,
$$

whence $J^{s, v}(f) \geq W(s, v)$. Thus, taking the infimum over $\quad{ }_{1485}$ all $f \in L^{2}(s, T ; F)$,

$$
W(s, v) \leq U(s, v) \quad \forall(s, v) \in(0, T) \times D(A) .
$$

Now, suppose there is a control function $f_{*} \in L^{2}(s, T ; F) \quad 1488$ such that, for all $t \in[s, T]$,

$$
\begin{array}{r}
\left\langle\partial_{\nu} W\left(t, u_{*}(t)\right), B f_{*}(t)\right\rangle+L\left(t, u_{*}(t), f_{*}(t)\right) \\
=\mathcal{H}\left(t, u_{*}(t), \partial_{v} W\left(t, u_{*}(t)\right)\right),
\end{array}
$$

where $u_{*}(\cdot)=u_{f_{*}}^{s, v}(\cdot)$. Then, from (71) and (73) it follows that

$$
\frac{\mathrm{d}}{\mathrm{d} t} W\left(t, u_{*}(t)\right)=-L(t, u(t), f(t)),
$$

whence

$$
W(s, v)=J^{s, v}\left(f_{*}\right) \geq U(s, v) .
$$

From the above inequality and (72) it follows that 1499 $W(s, v)=U(s, v)$ for all $(s, v) \in(0, T) \times D(A)$, hence for all $(s, v) \in(0, T) \times H$ since $D(A)$ is dense in $H$. So, $f_{*}$ is an optimal control.

Note 2 The above considerations lead to the following procedure to obtain optimal an optimal trajectory:

- find a smooth solution of the Hamilton-Jacobi equation; 
1525 and

$1544\left\{\begin{array}{cc}\partial_{s} W(s, v)+\left\langle A v, \partial_{v} W(s, v)\right\rangle+\langle M(s) v, v\rangle \\ -\frac{1}{4}\left\langle B N^{-1}(s) B^{*} \partial_{v} W(s, v), \partial_{v} W(s, v)\right\rangle=0 \\ \quad \forall(s, v) \in(0, T) \times D(A) \\ w(T, v)=\langle D v, v\rangle \quad \forall v \in H\end{array}\right.$ $f(t, v)$ such that

$$
\begin{aligned}
\left\langle\partial_{v} W(t, v), B f(t, v)\right\rangle+L(t, v, f(t, v)) & \\
& =\mathcal{H}\left(t, v, \partial_{v} W(t, v)\right)
\end{aligned}
$$

- solve the so-called closed loop equation

$$
\left\{\begin{array}{l}
u^{\prime}(t)=A u(t)+B f(t, u(t)) \quad t \in[s, T] \\
u(s)=v
\end{array}\right.
$$

Notice that not only is trajectory $u$ optimal, but the corresponding control $f$ is given in feedback form as well.

\section{Linear Quadratic Optimal Control}

One of the most successful applications of DP is the socalled Linear Quadratic optimal control problem. Consider problem (68)-(69) with costs $L$ and $\ell$ given by

$$
\begin{aligned}
L(t, u, \phi)=\langle M(t) u, u\rangle & +\langle N(t) \phi, \phi\rangle \\
\forall(T, u, \phi) & \in[0, T] \times H \times F
\end{aligned}
$$

$$
\ell(u)=\langle D u, u\rangle \quad \forall u \in H,
$$

where

- $M:[0, T] \rightarrow \mathcal{L}(H)$ is continuous, $M(t)$ is symmetric and $\langle M(t) u, u\rangle \geq 0$ for every $(t, u) \in[0, T] \times H$;

- $N:[0, T] \rightarrow F$ is continuous, $N(t)$ is symmetric and $\langle N(t) \phi, \phi\rangle \geq c_{0}|\phi|^{2}$ for every $(t, \phi) \in[0, T] \times F$ and some constant $c_{0}>0$;

- $D \in \mathcal{L}(H)$ is symmetric and $\langle D u, u\rangle \geq 0$ for every $u \in H$.

Then, assumptions (63) and (64) are satisfied. So, a solution to (68)-(69) does exist. Moreover, it is unique because of the strict convexity of functional $J^{s, v}$.

In order to apply DP, one computes the Hamiltonian

$$
\begin{aligned}
\mathcal{H}(t, u, p) & =\min _{\phi \in F}[\langle p, B \phi\rangle+\langle M(t) u, u\rangle+\langle N(t) \phi, \phi\rangle] \\
& =\langle M(t) u, u\rangle-\frac{1}{4}\left\langle B N^{-1}(t) B^{*} p, p\right\rangle,
\end{aligned}
$$

where the above minimum is attained at

$$
\phi_{*}(t, p)=-\frac{1}{2} N^{-1}(t) B^{*} p .
$$

Therefore, the Hamilton-Jacobi equation associated to the problem is

It is quite natural to search a solution of the above problem in the form

$$
W(s, v)=\langle P(s) v, v\rangle \quad \forall(s, v) \in[0, T] \times H,
$$

with $P:[0, T] \rightarrow \mathcal{L}(H)$ continuous, symmetric and such that $\langle P(t) u, u\rangle \geq 0$. Substituting into the Hamilton-Jacobi equation yields

$\left\{\begin{array}{c}\left\langle P^{\prime}(s) v, v\right\rangle+\left\langle\left[A^{*} P(s)+P(s) A\right] v, v\right\rangle+\langle M(s) v, v\rangle \\ -\left\langle B N^{-1}(s) B^{*} P(s) v, P(s) v\right\rangle=0 \\ \forall(s, v) \in(0, T) \times D(A) \\ \langle P(T) v, v\rangle=\langle D v, v\rangle \quad \forall v \in H\end{array}\right.$

Therefore, $P$ must be a solution of the so-called Riccati 1552 equation

$$
\left\{\begin{array}{l}
P^{\prime}(s)+A^{*} P(s)+P(s) A+M(s) \\
\quad-P(s) B N^{-1}(s) B^{*} P(s)=0 \\
P(T)=D
\end{array} \quad \forall s \in(0, T)\right.
$$

Once a solution $P(\cdot)$ the Riccati equation is known, the procedure described in Note 2 can be applied. Indeed, recalling (74) and the fact that $\partial_{v} W(t, v)=2 P(t) v$, one concludes that $f(t, v)=-N^{-1}(t) B^{*} P(t) v$ is a feedback law. So, solving the closed loop equation

$$
\left\{\begin{array}{l}
u^{\prime}(t)=\left[A-B N^{-1}(t) B^{*} P(t)\right] u(t) \quad t \in(s, T) \\
u(s)=v
\end{array}\right.
$$

one obtains the unique optimal trajectory of problem (68)-(69)

In sum, by DP one reduces the original Linear Quadratic optimal control problem to the problem of finding the solution of the Riccati equation, which is easier to solve than the Hamilton-Jacobi equation.

\section{Bibliographical Comments}

Different variants of the Riccati equation have been successfully studied by several authors in connection with different state equations and cost functionals, including boundary control problems and problems for other functional equations, see [22,79] and the references therein. Sometimes, the solution of the Riccati equation related to a linearized model provides feedback stabilization for nonlinear problems as in [104].

Unfortunately, the DP method is hard to implement for general optimal control problems, because of several obstructions: nonsmoothness of solutions to HamiltonJacobi equations, selection problems that introduce discontinuities, unboundedness of the coefficients, numerical complexity. Besides the Linear Quadratic case, the 
so-called Linear Convex case is the other example that can be studied by DP under fairly general conditions, see [14]. For nonlinear optimal control problems some of the above difficulties have been overcome extending the notion of viscosity solutions to infinite dimensional spaces, see $[45,46,47,48,49]$, see also $[28,29,30,31,32,33]$ and [112]. Nevertheless, finding additional ideas to make a generalized use of DP for distributed parameter systems possible, remains a challenging problem for the next generations.

\section{Future Directions}

In addition to all considerations spread all over this article on promising developments of recent-as well as established-research lines, a few additional topics deserve to be mentioned.

The one subject that has received the highest attention, recently, is that of numerical approximation of control problems, from the point of view of both controllability and optimal control. Here the problem is that, due to high frequency spurious numerical solutions, stable algorithms for solving initial-boundary value problems do not necessarily yield convergent algorithms for computing controls. This difficulty is closely related to the existence of concentrated numerical solutions that escape the observation mechanisms. Nevertheless, some interesting results have been obtained so far, see, e. g., $[124,125]$.

Several interesting results for nonlinear control problems have been obtained by the return method, developed initially by Coron [42] for a stabilization problem. This and other techniques have then been applied to fluid models ([68,69]), the Korteweg-de Vries equation ([105,106,107], and Schrödinger type equations ([19]), see also [43] and the references therein. It seems likely that these ideas, possibly combined with other techniques like Carleman estimates as in [70], will lead to new exiting results in the years to come.

A final comment on null controllability for degenerate parabolic equations is in order. Indeed, many problems that are relevant for applications are described by parabolic equation equations in divergence form

$\partial_{t} u=\nabla \cdot(A(x) \nabla u)+b(x) \cdot \nabla u+c(t, x) u+f \quad$ in $\quad Q_{T}$,

or in the general form

$\partial_{t} u=\operatorname{Tr}\left[A(x) \nabla^{2} u\right]+b(x) \cdot \nabla u+c(t, x) u+f \quad$ in $\quad Q_{T}$,

where $A(x)$ is a symmetric matrix, positive definite in $\Omega$ but possibly singular on $\Gamma$. For instance, degenerate parabolic equations arise in fluid dynamics as suitable transformations of the Prandtl equations, see, e. g., [94]. They can also be obtained as Kolmogorov equations of diffusions processes on domains that are invariant for stochastic flows, see, e. g., [52]. The latter interpretation explains why they have been applied to biological problems, such as gene frequency models for population genetics (see, e. g., the Wright-Fischer model studied in [111]).

So far, null controllability properties of degenerate parabolic equations have been fully understood only in dimension one: for some kind of degeneracy, null controllability holds true (see [36] and [9]), but, in general, one can only expect regional null controllability (see [35]). Since very little is known on null controllability for degenerate parabolic equations in higher space dimensions, it is conceivable that such a topic will provide interesting problems for future developments.

\section{Bibliography}

1. Alabau $F$ (1999) Stabilisation frontière indirecte de systèmes faiblement couplés. C R Acad Sci Paris Sér I 328:1015-1020

2. Alabau $F$ (2002) Indirect boundary stabilization of weakly coupled systems. SIAM J Control Optim 41(2):511-541

3. Alabau-Boussouira F (2003) A two-level energy method for indirect boundary observability and controllability of weakly coupled hyperbolic systems. SIAM J Control Optim 42(3):871906

4. Alabau-Boussouira F (2004) A general formula for decay rates of nonlinear dissipative systems. C R Math Acad Sci Paris 338:35-40

5. Alabau-Boussouira F (2005) Convexity and weighted integral inequalities for energy decay rates of nonlinear dissipative hyperbolic systems. Appl Math Optim 51(1):61-105

6. Alabau-Boussouira F (2006) Piecewise multiplier method and nonlinear integral inequalities for Petrowsky equations with nonlinear dissipation. J Evol Equ 6(1):95-112

7. Alabau-Boussouira F (2007) Asymptotic behavior for Timoshenko beams subject to a single nonlinear feedback control. NoDEA 14(5-6):643-669

8. Alabau F, Cannarsa P, Komornik V (2002) Indirect interna damping of coupled systems. J Evol Equ 2:127-150

9. Alabau-Boussouira F, Cannarsa P, Fragnelli G (2006) Carleman estimates for degenerate parabolic operators with applications to null controllability. J Evol Equ 6:161-204

10. Alabau-Boussouira F, Cannarsa P, Sforza D (2008) Decay estimates for second order evolution equations with memory. J Funct Anal 254(5):1342-1372

11. Ammari K, Tucsnak M (2001) Stabilization of second order evolution equations by a class of unbounded feedbacks. ESAIM Control Optim Calc Var 6:361-386

12. Ammar-Khodja F, Bader A, Benabdallah A (1999) Dynamic stabilization of systems via decoupling techniques. ESAIM Control Optim Calc Var 4:577-593

13. Barbu V (2003) Feedback stabilization of Navier-Stokes equations. ESAIM Control Optim Calc Var 9:197-206

14. Barbu V Da Prato G (1983) Hamilton Jacobi equations in Hilbert spaces. Pitman, London 
15. Barbu V, Lasiecka I, Triggiani R (2006) Tangential boundary stabilization of Navier-Stokes equations. Mem Amer Math Soc 181(852):CE2

16. Barbu V, Triggiani R (2004) Internal stabilization of NavierStokes equations with finite-dimensional controllers. Indiana Univ Math J 53(5):1443-1494

17. Bardos C, Lebeau G, Rauch R (1992) Sharp sufficient conditions for the observation, control, and stabilization of waves from the boundary. SIAM J Control Optim 30:1024-1065

18. Bátkai A, Engel KJ, Prüss J, Schnaubelt R (2006) Polynomial stability of operator semigroups. Math Nachr 279(1314):1425-1440

19. Beauchard K (2005) Local controllability of a 1-D Schödinger equation. J Math Pures Appl 84(7):851-956

20. Bellman R (1957) Dynamic Programming. Princeton University Press, Princeton

21. Benabdallah A, Dermenjian Y, Le Rousseau J (2007) Carleman estimates for the one-dimensional heat equation with a discontinuous coefficient and applications to controllability and an inverse problem. J Math Anal Appl 336(2):865-887

22. Bensoussan A, Da Prato G, Delfour MC, Mitter SK (1993) Representation and Control of Infinite Dimensional Systems. Systems and Control: Foundations and applications, Birkhäuser, Boston

23. Beyrath A (2001) Stabilisation indirecte localement distribué de systèmes faiblement couplés. C R Acad Sci Paris Sér I Math 333(5):451-456

24. Beyrath A (2004) Indirect linear locally distributed damping of coupled systems. Bol Soc Parana Mat 22(2):17-34CE3

25. Burq N, Gérard P (1997) Condition nécessaire et suffisante pour la contrôlabilité exacte des ondes. C R Acad Sci Paris Sér I Math 325(7):749-752

26. Burq N, Hitrik M (2007) Energy decay for damped wave equations on partially rectangular domains. Math Res Lett 14(1):35-47

27. Burq N, Lebeau G (2001) Mesures de défaut de compacité, application au système de Lamé. Ann Sci École Norm Sup (4) 34(6):817-870 CE3

28. Cannarsa P (1989) Regularity properties of solutions to Hamilton-Jacobi equations in infinite dimensions and nonlinear optimal control. Diff Integral Equ 2:479-493

29. Cannarsa P, Da Prato G (1990) Some results on non-linear optimal control problems and Hamilton-Jacobi equations in infinite dimensions. J Funct Anal 90:27-47

30. Cannarsa P, Gozzi F, Soner HM (1991) A boundary value problem for Hamilton-Jacobi equations in Hilbert spaces. Applied Math Optim 24:197-220

31. Cannarsa P, Gozzi F, Soner HM (1993) A dynamic programming approach to nonlinear boundary control problems of parabolic type. J Funct Anal 117:25-61

32. Cannarsa P, Di Blasio G (1995) A direct approach to infinite dimensional Hamilton-Jacobi equations and applications to convex control with state constraints. Differ Integral Equ 8:225-246

33. Cannarsa P, Tessitore ME (1996) Infinite dimensional Hamilton-Jacobi equations and Dirichlet boundary control problems of parabolic type. SIAM J Control Optim 34:1831-1847

34. Cannarsa P, Komornik V, Loreti P (2002) One-sided and internal controllability of semilinear wave equations with infinitely iterated logarithms. Discret Contin Dyn Syst 8(3):745-756
35. Cannarsa P, Martinez P, Vancostenoble J (2004) Persistent regional null controllability for a class of degenerate parabolic equations. Commun Pure Appl Anal 3(4):607-635

36. Cannarsa P, Martinez P, Vancostenoble (2005) Null Controllability of degenerate heat equations. Adv Differ Equ 10(2):153190

37. Cavalcanti MM, Oquendo HP (2003) Frictional versus viscoelastic damping in a semilinear wave equation. SIAM J Control Optim 42:1310-1324

38. Chen G (1981) A note on boundary stabilization of the wave equation. SIAM J Control Optim 19:106-113

39. Chueshov I, Lasiecka I, Toundykov D (2008) Long-term dynamics of semilinear wave equation with nonlinear localized interior damping and a source term of critical exponent. Discret Contin Dyn Syst 20(3):459-509

40. Conrad F, Rao B (1993) Decay of solutions of the wave equation in a star-shaped domain with nonlinear boundary feedback. Asymptot Anal 7:159-177

41. Conrad F, Pierre (1994) Stabilization of second order evolution equations by unbounded nonlinear feedback. Ann Inst H Poincaré Anal Non Linéaire 11(5):485-515, Asymptotic Anal 7:159-177

42. Coron JM (1992) Global asymptotic stabilization for controllable systems without drift. Math Control Signals Syst 5(3):295-312

43. Coron JM (2007) Control and nonlinearity. American Mathematical Society, Mathematical surveys and monographs vol 136CE4

44. Coron JM, Trélat E (2004) Global steady-state controllability of one-dimensional semilinear heat equations. SIAM J Control Optim 43(2):549-569

45. Crandall MG, Lions PL (1985) Hamilton Jacobi equation in infinite dimensions I: Uniqueness of viscosity solutions. J Funct Anal 62:379-396

46. Crandall MG, Lions PL (1986) Hamilton Jacobi equation in infinite dimensions II: Existence of viscosity solutions. J Funct Anal 65:368-425

47. Crandall MG, Lions PL (1986) Hamilton Jacobi equation in infinite dimensions III. J Funct Anal 68:214-247

48. Crandall MG, Lions PL (1990) Hamilton Jacobi equation in infinite dimensions IV: Hamiltonians with unbounded linear terms. J Funct Anal 90:237-283

49. Crandall MG, Lions PL (1991) Hamilton Jacobi equation in infinite dimensions V: Unbounded linear terms and B-continuous solutions. J Funct Anal 97:417-465

50. Curtain RF, Weiss G (1989) Well posedness of triples of operators (in the sense of linear systems theory). Control and Estimation of Distributed Parameter Systems (Vorau, 1988). Internat. Ser. Numer. Math., vol. 91, Birkhäuser, Basel

51. Curtain RF, Zwart H (1995) An introduction to infinite-dimensional linear systems theory. Texts in Applied Mathematics, vol 21. Springer, New York

52. Da Prato G, Frankowska H (2007) Stochastic viability of convex sets. J Math Anal Appl 333(1):151-163

53. Dafermos CM(1970) Asymptotic stability inviscoelasticity. Arch Ration Mech Anal 37:297-308

54. Dafermos CM (1970) An abstract Volterra equation with applications to linear viscoelasticity. J Differ Equ 7:554-569

55. Engel KJ, R Nagel R (2000) One-parameter semigroups for linear evolution equations. Springer, New York 
56. Eller M, Lagnese JE, Nicaise S (2002) Decay rates for solutions of a Maxwell system with nonlinear boundary damping. Comp Appl Math 21:135-165

57. Fabre C, Puel J-P, Zuazua E (1995) Approxiamte controllability of the semilinear heat equation. Proc Roy Soc Edinburgh Sect A 125(1):31-61

58. Fattorini HO, Russell DL (1971) Exact controllability theorems for linear parabolic equations in one space dimension. Arch Rat Mech Anal 4:272-292

59. Fattorini HO (1998) Infinite Dimensional Optimization and Control theory. Encyclopedia of Mathematics and its Applications, vol 62. Cambridge University Press, Cambridge

60. Fattorini HO (2005) Infinite dimensional linear control systems. North-Holland Mathematics Studies, vol 201. Elsevier Science B V, Amsterdam

61. Fernández-Cara E, Zuazua E (2000) Null and approximate controllability for weakly blowing up semilinear heat equations. Ann Inst H Poincaré Anal Non Linéaire 17(5):583-616

62. Fernández-Cara E, Zuazua E (2000) The cost approximate controllability for heat equations: The linear case. Adv Differ Equ 5:465-514

63. Fernández-Cara E, Guerrero S, Imanuvilov OY, Puel J-P (2004) Local exact controllability of the Navier-Stokes system. J Math Pures Appl 83(9-12):1501-1542

64. Fursikov A (2000) Optimal control of distributed systems. Theory and applications. Translation of Mathematical Monographs, vol 187. American Mathematical Society, Providence, RI. Translated from the 1999 Russian original by Tamara RozhkovskayaCE4

65. Fursikov A, Imanuvilov OY (1996) Controllability of evolution equations. Lecture Notes, Research Institute of Mathematics, Seoul National University, Seoul

66. Gibson JS (1980) A note on stabilization of infinite dimensional linear oscillators by compact linear feedback. SIAM J Control Optim 18:311-316

67. Giorgi C, Naso MG, Pata V (2005) Energy decay of electromagnetic systems with memory. Math Models Methods Appl Sci 15(10):1489-1502

68. Glass O (2000) Exact boundary controllability of 3-D Euler equation. ESAIM Control Optim Calc Var 5:1-44

69. Glass O (2007) On the controllability of the 1-D isentropic Euler equation. J Eur Math Soc (JEMS) 9(3):427-486

70. Guerrero S (2007) Local controllability to the trajectories of the Navier-Stokes system with nonlinear Navier-slip boundary conditions. ESAIM Control Optim Calc Var 12(3):484-544

71. Haraux A (1978) Semi-groupes linéaires et équations d'évolution linéaires périodiques. Publication du Laboratoire d'Analyse Numérique no 78011. Université Pierre et Marie Curie, Paris

72. Haraux A (1989) Une remarque sur la stabilisation de certains systèmes du deuxième ordre en temps. Portugal Math 46(3):245-258

73. Ho LF (1986) Observabilité frontière de l'équation des ondes. C R Acad Sci Paris Sér I Math 302(12):443-446

74. Komornik V (1994) Exact Controllability and Stabilization. The Multiplier Method. Collection RMA, vol 36. Masson-John Wiley, Paris-Chicester

75. Komornik V, Loreti P (2005) Fourier series in control theory. Springer, New York
76. Komornik V, Zuazua E (1990) A direct method for the boundary stabilization of the wave equation. J Math Pures Appl 69:33-54

77. Lasiecka I, Lions J-L, Triggiani R (1986) Nonhomogeneous boundary value problems for second order hyperbolic operators. J Math Pures Appl 65(2):149-192

78. Lasiecka I, Tataru D (1993) Uniform boundary stabilization of semilinear wave equation with nonlinear boundary damping. Differ Integral Equ 8:507-533

79. Lasiecka I, Triggiani R (1991) Differential and algebraic Riccati equations with application to boundary/point control problems: continuous theory and approximation theory. Lecture Notes in Control \& Inform Sci, vol 164. Springer, Berlin

80. Lasiecka I, Triggiani R (2000) Control theory for partial differential equations: continuous and approximation theories. I. Encyclopedia of Mathematics and its Applications, vol 74. Cambridge University Press, Cambridge

81. Lasiecka I, Triggiani R (2000) Control theory for partial differential equations: continuous and approximation theories. II. Encyclopedia of Mathematics and its Applications, vol 75. Cambridge University Press, Cambridge

82. Le Rousseau J (2007) Carleman estimates and controllability results for the one-dimensional heat equation with BV coefficients. J Differ Equ 233(2):417-447

83. Lebeau G, Robbiano L (1995) Exact control of the heat equation. Comm Partial Differ Equ 20(1-2):335-356

84. Li X, Yong J (1995) Optimal control of infinite dimensional systems. Systems \& Control: Foundations \& Applications. Birkhäuser, Boston

85. Lions J-L (1971) Optimal control of systems governed by partial differential equations. Springer, New-York

86. Lions J-L (1988) Contrôlabilité exacte et stabilisation de systèmes distribués I-II. Masson, Paris

87. Liu K (1997) Locally distributed control and damping for the conservative systems. SIAM J Control Optim 35:1574-1590

88. Liu Z, Zheng S (1999) Semigroups associated with dissipative systems. Chapman Hall CRC Research Notes in Mathematics, vol 398. Chapman Hall/CRC, Boca Raton

89. Liu WJ, Zuazua E (1999) Decay rates for dissipative wave equations. Ric Mat 48:61-75

90. Liu Z, Rao R (2007) Frequency domain approach for the polynomial stability of a system of partially damped wave equations. J Math Anal Appl 335(2):860-881

91. Londen SO, Petzeltová H, Prüss J (2003) Global well-posedness and stability of a partial integro-differential equation with applications to viscoelasticity. J Evol Equ 3(2):169-201

92. Loreti $P$, Rao B (2006) Optimal energy decay rate for partially damped systems by spectral compensation. SIAM J Control Optim 45(5):1612-1632

93. Martinez P (1999) A new method to obtain decay rate estimates for dissipative systems with localized damping. Rev Mat Complut 12:251-283

94. Martinez P, Raymond J-P, Vancostenoble J (2003) Regional null controllability for a linearized Crocco type equation. SIAM J Control Optim 42(2):709-728

95. Miller L (2002) Escape function conditions for the observation, control, and stabilization of the wave equation. SIAM J Control Optim 41(5):1554-1566

96. Muñoz Rivera JE, Peres Salvatierra A (2001) Asymptotic be- 
haviour of the energy in partially viscoelastic materials. Quart Appl Math 59:557-578

97. Muñoz Rivera JE (1994) Asymptotic behaviour in linear viscoelasticity. Quart Appl Math 52:628-648

98. Nakao M (1996) Decay of solutions of the wave equation with a local nonlinear dissipation. Math Ann 305:403-417

99. Pazy A (1968) Semigroups of linear operators and applications to partial differential equations. Springer Berlin

100. Pontryagin LS (1959) Optimal regulation processes. Uspehi Mat Nauk 14(1):3-20

101. Pontryagin LS, Boltyanskii VG, Gamkrelidze RV, Mishchenko EF (1962) The mathematical theory of optimal processes. Interscience Publishers John Wiley \& Sons, Inc., New YorkLondon, translated from the Russian by Trirogoff KN, edited by Neustadt LW

102. Propst G, Prüss J (1996) On wave equation with boundary dissipation of memory type. J Integral Equ Appl 8:99-123

103. Prüss J (1993) Evolutionary integral equations and applications. Monographs in Mathematics, vol 87. Birkhäuser Verlag, Basel

104. Raymond J-P (2006) Feedback boundary stabilization of the two-dimensional Navier-Stokes equations. SIAM J Control Optim 45(3):790-828

105. Rosier L (1997) Exact boundary controllability for the Korteweg-de Vries equation on a bounded domain. ESAIM Control Optim Calc Var 2:33-55

106. Rosier L (2000) Exact boundary controllability for the linear Korteweg-de Vries equation on the half-line. SIAM J Control Optim 39(2):331-351

107. Rosier L, Zhang BY (2006) Global stabilization of the generalized Korteweg-de Vries equation posed on a finite domain. SIAM J Control Optim 45(3):927-956

108. Russell DL (1978) Controllability and stabilizability theorems for linear partial differential equations: recent progress and open questions. SIAM Rev 20(4):639-739

109. Russell DL (1993) A general framework for the study of indirect damping mechanisms in elastic systems. J Math Anal Appl 173(2):339-358

110. Salamon D (1987) Infinite-dimensional linear systems with unbounded control and observation: a functional analytic approach. Trans Am Math Soc 300(2):383-431

111. Shimakura N (1992) Partial differential operators of elliptic type. Translations of Mathematical Monographs, vol 99American Mathematical Society, Providence

112. Tataru D (1992) Viscosity solutions for the dynamic programming equations. Appl Math Optim 25:109-126

113. Tataru D (1994) A-priori estimates of Carleman's type in domains with boundary. J Math Pures Appl 75:355-387

114. Tataru D (1995) Boundary controllability for conservative P.D.E. Appl Math Optim 31:257-295

115. Tataru D (1996) Carleman estimates and unique continuation near the boundary for P.D.E.'s. J Math Pures Appl 75:367-408

116. Tataru D (1997) Carleman estimates, unique continuation and controllability for anizotropic PDE's. Contemporary Mathematics, vol 209. CE5 pp 267-279

117. de Teresa $L$ (2000) Insensitizing controls for a semilinear heat equation. Comm Partial Differ Equ 25:39-72

118. Vancostenoble J, Martinez P (2000) Optimality of energy estimates for the wave equation with nonlinear boundary velocity feedbacks. SIAM J Control Optim 39:776-797

119. Vancostenoble J (1999) Optimalité d'estimation d'énergie pour une équation des ondes amortie. C R Acad Sci Paris, 328, 1979 série I, pp 777-782

120. Vitillaro E (2002) Global existence for the wave equation with nonlinear boundary damping and source terms. J Differ Equ 186(1):259-298

121. Zabczyk J (1992) Mathematical control theory: an introduction. Birkhäuser, Boston

122. Zuazua E (1989) Uniform stabilization of the wave equation by nonlinear feedbacks. SIAM J Control Optim 28:265-268

123. Zuazua E (1990) Exponential decay for the semilinear wave equation with locally distributed damping. Comm Partial Differ Equ 15:205-235

124. Zuazua E (2006) Control and numerical approximation of the heat and wave equations. In: Sanz-Solé M, Soria J, Juan Luis V, Verdera J (eds) Proceedings of the International Congress of Mathematicians, vol I, II, III, European Mathematical Society, Madrid, pp 1389-1417

125. Zuazua E (2006) Controllability and observability of partial differential equations: Some results and open problems. In: Dafermos CM, Feireisl E (eds) Handbook of differential equations: evolutionary differential equations, vol 3. Elsevier/North-Holland, Amsterdam, pp 527-621 